\title{
Split Decomposition over an Abelian Group Part 1: Generalities
}

\author{
Andreas Dress $1,2 *$ \\ ${ }^{1}$ Department for Combinatorics and Geometry, CAS-MPG Partner Institute and Key Lab for \\ Computational Biology, Shanghai Institutes for Biological Sciences, Chinese Academy of \\ Sciences, 320 Yue Yang Road, Shanghai 200031, P.R.China \\ ${ }^{2}$ Max Planck Institute for Mathematics in the Sciences, Inselstrasse 22 -26, D 04103 Leipzig, \\ Germany \\ dress@ sibs.ac.cn,dress@mis.mpg.de
}

Received April 30, 2006

AMS Subject Classification: 05C05, 92D15

\begin{abstract}
Split-decomposition theory deals with relations between $\mathbb{R}$-valued split systems and metrics. Here, we generalize (parts of) this theory, considering group-valued split systems that take their values in an arbitrary abelian group, and replacing metrics by certain, appropriately defined maps (some of which appear to exhibit a decidedly algebraic flavour). In the second and the third parts of this series of papers, the main results of split-decomposition theory will be established within this conceptual framework.
\end{abstract}

Keywords: splits, split decomposition, split systems, set systems, group-valued split systems, group-valued set systems, phylogenetic analysis, metrics, bilinear maps, groupoid

\section{Introduction}

A standard approach in phylogenetic analysis is to model the desired result, i.e., the branching pattern of species evolution, in terms of a system of weighted, pairwise compatible splits. Furthermore, to find this system, one usually supposes that some sort of traces have been left by the branching process in question that are detectable by investigating present-day species and have preserved sufficient information to allowing us to reconstruct the required branching pattern.

Most often, these traces are represented by estimates, one for each pair $x, y$ of species under consideration, of the total amount $D(x, y)$ of genetic change that separates Species $x$ from Species $y$ - see, for instance, [26] in which this kind of approach has been applied, for the first time, to a family of protein sequences, viz., the family of Cytochrome $\mathrm{C}$ sequences of altogether 20 eukaryotic species including fungi, plants,

\footnotetext{
* Partly supported by the Science Technology Commission of Shanghai Municipality (Grant 06ZR14048).
} 
and animals, see also e.g., $[4,12]$ for suggestions of other approaches towards using such standard distance data.

However, other types of traces have also been taken into consideration as they, sometimes, appear to be easier to handle. For example, given a further fixed species $z$ - if possible, an appropriately chosen outgroup taxon relative to the set taxa under investigation - one may estimate the total amount $D_{z}(x, y)$ of genetic change that separates this outgroup species $z$ from the latest common precursor of Species $x$ and Species $y$ - or, rather, from that species on the imagined evolutionary path connecting Species $x$ and Species $y$ that is closest to Species $z$ (cf. [22-24], see also [17] for a recent survey and further references regarding this procedure).

Alternatively, one may also consider, for any three species $a, b$, and $c$, the total amount $D(a, b, c)$ of phylogenetic diversity represented by these three species (cf. [9, 27, 28, 30,33], and [19] for a recent discussion of these approaches).

And one may consider, for any four given species $x, y, u, v$, the difference $D_{y}(x, u)$ $-D_{y}(x, v)$ - a "four-variate" trace that was used recently by Backelin and Linusson in their investigation of parity splits (cf. [2]).

In the first part of the series of papers, we will investigate the relationship between the various traces that one can associate to any given weighted split system. To this end, we will generalize (parts of) split-decomposition theory which, in its original form as developed in [4] and described for the non-mathematically oriented audience in [6], deals with positively weighted split systems, relating their "standard" traces to metrics. Furthermore, to recover all of the (mathematical) results obtained in the various papers quoted above dealing with trace-based phylogenetic reconstruction, and taking into account in particular that Backelin' and Linusson's work deals with the group of order 2 (and not at all with the additive group of real numbers), we'll follow the approach pioneered already in [8] and also adopted in [19]: That is, we will allow our split systems to attain their values in an arbitrary abelian group $\mathcal{A}$, and we will, of course, do the same for the various groups encompassing all imaginable traces to be investigated here.

More specifically, given a non-empty set $X$, an element $z \in X$, and an (additively written) abelian group $\mathcal{A}$ with neutral element $0=0_{\mathcal{A}}$, we will consider

(1) the group $\mathcal{S}^{*}(X \mid \mathcal{A})$ consisting of all maps $\Sigma$ of finite support ${ }^{\dagger}$ from the set $\mathcal{S}(X)$ of all splits of the set $X$ (i.e., all unordered pairs $\{A, B\}$ of disjoint subsets $A, B$ of $X$ for which $A \cup B=X$ holds $)$ into the group $\mathcal{A}$ for which $\Sigma(\{X, \emptyset\})=0$ holds — modeling, in a rather abstract way, the branching (or, better, the "separation") patterns of species evolution,

and the following abelian groups whose elements can be viewed — in an equally abstract way - as constructs modeling the various types of traces that these branching patterns may be supposed to have left:

(2) the group $\mathcal{P}_{\leq 2}(X \mid \mathcal{A})$ consisting of all maps

$$
\Pi:\left(\begin{array}{c}
X \\
\leq 2
\end{array}\right) \rightarrow \mathcal{A}:\{a, b\} \mapsto \Pi(a b)
$$

\footnotetext{
${ }^{\dagger}$ Recall that the support $\operatorname{supp}(f)$ of any map $f$ from a set $I$ into a group $\mathcal{A}$ is the set $\{i \in I: f(i) \neq 0\}$.
} 
from the set $\left(\begin{array}{c}X \\ \leq 2\end{array}\right)$ of all non-empty subsets of $X$ of cardinality at most 2 into the group $\mathcal{A}$ and its two subgroups

$$
\begin{aligned}
\mathcal{P}_{(2)}(X \mid \mathcal{A}):=\left\{\Pi \in \mathcal{P}_{\leq 2}(X \mid \mathcal{A}): \forall_{a, b, c \in X} \Pi(a)=0,\right. \\
\Pi(a b)+\Pi(b c)+\Pi(c a) \in 2 \mathcal{A}\}
\end{aligned}
$$

(with $\Pi(a)=\Pi(\{a\})$ and $2 \mathcal{A}:=\{2 \alpha: \alpha \in \mathcal{A}\}$, of course), and

$$
\mathcal{P}_{\leq 2}(X, z \mid \mathcal{A}):=\left\{\Pi \in \mathcal{P}_{\leq 2}(X \mid \mathcal{A}): \forall_{a \in X} \Pi(a z)=0\right\},
$$

(3) the group $\mathcal{P}_{\leq 3}(X \mid \mathcal{A})$ consisting of all maps

$$
\Psi:\left(\begin{array}{c}
X \\
\leq 3
\end{array}\right) \in \mathcal{A}:\{a, b, c\} \mapsto \Psi(a b c)
$$

from the set $\left(\begin{array}{c}X \\ \leq 3\end{array}\right)$ of all non-empty subsets of $X$ of cardinality at most 3 into the group $\mathcal{A}$, and its subgroup $\mathcal{P}_{(3)}(X \mid \mathcal{A})$ consisting of all maps $\Psi$ in $\mathcal{P}_{\leq 3}(X \mid \mathcal{A})$ for which (with $\Psi(x):=\Psi(\{x\}), \Psi(x y):=\Psi(\{x, y\})$, and $\Psi(x y z):=\Psi(\{x y z\})$ for all $x, y, z \in X)$ one has $\Psi(a)=0$ for all $a \in X$ and

$$
\sum_{I \in\left(\begin{array}{c}
\{1,2,3,4\} \\
3
\end{array}\right)} \Psi\left(\left\{a_{i}: i \in I\right\}\right)=\sum_{J \in\left(\begin{array}{c}
\{1,2,3,4\} \\
2
\end{array}\right)} \Psi\left(\left\{a_{j}: j \in J\right\}\right)
$$

for all $a_{1}, a_{2}, a_{3}, a_{4} \in X$ (or, equivalently,

$$
\begin{aligned}
\Psi(a b c)+\Psi(b c d)+\Psi(c d a)+\Psi(d a b)= & \Psi(a b)+\Psi(a c)+\Psi(a d)+\Psi(b c) \\
& +\Psi(b d)+\Psi(c d)
\end{aligned}
$$

for all $a, b, c, d \in X)$,

(4) and the group $\mathcal{L}_{2}(X \mid \mathcal{A})$ consisting of all "bilinear" symmetric maps

$$
\Lambda: \mathcal{G}(X)^{2} \rightarrow \mathcal{A}:((x, y),(u, v)) \mapsto \Lambda(x y: u v)
$$

defined on the groupoid $\mathcal{G}(X)$ canonically associated with the set $X$ whose elements are the pairs $(x, y)$ of elements from $X$ while a product $(x, y) *(u, v)$ of any two such pairs $(x, y),(u, v)$ is defined if and only if $y=u$ holds in which case $(x, y) *(u, v)$ is defined as $(x, y) *(u, v):=(x, v)^{*}$. That is, we define $\mathcal{L}_{2}(X \mid \mathcal{A})$ to denote the subgroup of the group $\mathcal{A}^{\mathcal{G}(X)^{2}}$ consisting of all maps from $\mathcal{G}(X)^{2}$ into $\mathcal{A}$ that consists of all those maps $\Lambda \in \mathcal{A}^{\mathcal{G}(X)^{2}}$ for which

$$
\Lambda(x y: u v)+\Lambda(x y: v w)=\Lambda(x y: u w)=\Lambda(u w: x y)
$$

holds for all $x, y, u, v, w \in X$.

Note that the symmetric bilinear maps $\Lambda$ from $\mathcal{G}(X)^{2}$ into the group $\{ \pm 1\}$ of order 2 turned up quite naturally in Backelin' and Linusson's investigations of parity splits presented in [2].

\footnotetext{
F In terms of category theory, $\mathcal{G}(X)$ is nothing but the — obviously "groupoidal" — category whose objects form the set $X$ while there is exactly one morphism from $x$ to $y$, denoted by $(x, y)$, for any two elements $x, y \in X$.
} 
We will now consider various canonically defined group homomorphisms between the seven groups $\mathcal{S}^{*}(X \mid \mathcal{A}), \mathcal{P}_{\leq 2}(X \mid \mathcal{A}), \mathcal{P}_{(2)}(X \mid \mathcal{A}), \mathcal{P}_{\leq 2}(X, z \mid \mathcal{A}), \mathscr{P}_{\leq 3}(X \mid \mathcal{A})$, $\mathcal{P}_{(3)}(X \mid \mathcal{A})$, and $\mathcal{L}_{2}(X \mid \mathcal{A})$ introduced above.

To this end, we will denote, for any subset $\mathcal{R}$ of $\mathcal{S}(X)$, any two subsets $Y, Z$ of $X$, and any map $\Sigma$ of finite support from $\mathcal{S}(X)$ into $\mathcal{A}$,

(i) by

$$
\Sigma_{+}(\mathcal{R}):=\sum_{S \in \mathcal{R}} \Sigma(S)
$$

the sum over all those values that the map $\Sigma$ attains at the splits in $\mathcal{R}$,

(ii) by $\mathcal{R}(Y \mid Z)$ the set of all splits in $\mathcal{R}$ that separate $Y$ from $Z$, i.e., the set of all splits $S=\{A, B\}$ in $\mathcal{R}$ with, say, $Y \subseteq A$ and $Z \subseteq B$,

(iii) and by

$$
\Sigma_{+}(Y \mid Z):=\Sigma_{+}(\mathcal{S}(Y \mid Z))
$$

the sum over all the values that the map $\Sigma$ attains at the set

$$
\mathcal{S}(Y \mid Z):=(\mathcal{S}(X))(Y \mid Z)
$$

consisting of all splits of $X$ that separate $Y$ from $Z$, writing also $\Sigma_{+}\left(y_{1} \cdots y_{i} \mid z_{1} \cdots\right.$ $\left.z_{j}\right)$ instead of $\Sigma_{+}(Y \mid Z)$ in case $Y=\left\{y_{1}, \ldots, y_{i}\right\}$ and $Z=\left\{z_{1}, \ldots, z_{j}\right\}$ holds for some $y_{1}, \ldots, y_{i}, z_{1}, \ldots, z_{j} \in X$.

Using these notations (and still assuming that some fixed element $z \in X$ is given), we can now associate, to each $\Sigma \in \mathcal{S}^{*}(X \mid \mathcal{A})$, its four "traces"

(1) $\Sigma^{(2)}:\left(\begin{array}{c}X \\ \leq 2\end{array}\right) \rightarrow \mathcal{A}:\{a, b\} \mapsto \Sigma_{+}(a \mid b)$,

(2) $\Sigma^{(z)}:\left(\begin{array}{c}X \\ \leq 2\end{array}\right) \rightarrow \mathcal{A}:\{a, b\} \mapsto \Sigma_{+}(a b \mid z)$,

(3) $\Sigma^{(3)}:\left(\begin{array}{c}X \\ \leq 3\end{array}\right) \rightarrow \mathcal{A}:\{a, b, c\} \mapsto \Sigma_{+}(a b \mid c)+\Sigma_{+}(b c \mid a)+\Sigma_{+}(c a \mid b)$,

(4) $\Sigma^{(\mathrm{bil})}:\left(X^{2}\right)^{2} \rightarrow \mathcal{A}:((x, y),(u, v)) \mapsto \Sigma_{+}(x v \mid y u)-\Sigma_{+}(x u \mid y v)$.

Furthermore, we can associate,

(i) to every map $\Pi \in \mathcal{P}_{\leq 2}(X \mid \mathcal{A})$ and all $a, b, c, x, y, u, v \in X$, the terms

$$
\begin{aligned}
\Pi^{(2)}(a b) & :=\Pi(a)+\Pi(b)-2 \Pi(a b), \\
\Pi^{(z)}(a b) & :=\Pi(a b)+\Pi(z)-\Pi(a z)-\Pi(b z), \\
\Pi^{(3)}(a b c) & :=\Pi(a)+\Pi(b)+\Pi(c)-\Pi(a b)-\Pi(b c)-\Pi(c a), \\
\Pi^{(b i l)}(x y: u v) & :=\Pi(x v)+\Pi(y u)-\Pi(x u)-\Pi(y v),
\end{aligned}
$$


thus associating also, to every map $\Pi \in \mathcal{P}_{\leq 2}(X \mid \mathcal{A})$, the four maps

$$
\begin{aligned}
& \Pi^{(2)}:\left(\begin{array}{c}
X \\
\leq 2
\end{array}\right) \rightarrow \mathcal{A}:\{a, b\} \mapsto \Pi^{(2)}(a b) \\
& \Pi^{(z)}:\left(\begin{array}{c}
X \\
\leq 2
\end{array}\right) \rightarrow \mathcal{A}:\{a, b\} \mapsto \Pi^{(z)}(a b), \\
& \Pi^{(3)}:\left(\begin{array}{c}
X \\
\leq 3
\end{array}\right) \rightarrow \mathcal{A}:\{a, b, c\} \mapsto \Pi^{(3)}(a b c), \\
& \Pi^{(\mathrm{bil})}:\left(X^{2}\right)^{2} \rightarrow \mathcal{A}:((x, y),(u, v)) \mapsto \Pi^{(\mathrm{bil})}(x y: u v),
\end{aligned}
$$

(ii) to every map $\Psi \in \mathcal{P}_{\leq 3}(X \mid \mathcal{A})$, and all $a, b, z, x, y, u, v \in X$, the terms

$$
\begin{aligned}
\Psi^{(2)}(a b) & :=\Psi(a b), \\
\Psi^{(z)}(a b) & :=\Psi(a b z)-\Psi(a b), \\
\Psi^{(\mathrm{bil})}(x y: u v) & :=\Psi(x y v)-\Psi(x v)-\Psi(x y u)+\Psi(x u),
\end{aligned}
$$

thus associating also, to every map $\Psi \in \mathcal{P}_{\leq 3}(X \mid \mathcal{A})$, the three maps

$$
\begin{aligned}
& \Psi^{(2)}:\left(\begin{array}{c}
X \\
\leq 2
\end{array}\right) \rightarrow \mathcal{A}:\{a, b\} \mapsto \Psi^{(2)}(a b), \\
& \Psi^{(z)}:\left(\begin{array}{c}
X \\
\leq 2
\end{array}\right) \rightarrow \mathcal{A}:\{a, b\} \mapsto \Psi^{(z)}(a b), \\
& \Psi^{(\mathrm{bil})}:\left(X^{2}\right)^{2} \rightarrow \mathcal{A}:((x, y),(u, v)) \mapsto \Psi^{(\mathrm{bil})}(x y: u v),
\end{aligned}
$$

(iii) and, to every map $\Lambda \in \mathcal{A}^{\left(X^{2}\right)^{2}}$ and all $a, b, c, z \in X$, the terms

$$
\begin{aligned}
\Lambda^{(2)}(a b) & :=\Lambda(a b: b a), \\
\Lambda^{(z)}(a b) & :=\Lambda(a z: z b), \\
\Lambda^{(3)}(a b c) & :=\Lambda(a b: b c)+\Lambda(b c: c a)+\Lambda(c a: a b),
\end{aligned}
$$

thus associating also, to every map $\Lambda \in \mathcal{A}^{\left(X^{2}\right)^{2}}$, the three maps

$$
\begin{aligned}
& \Lambda^{(2)}:\left(\begin{array}{c}
X \\
\leq 2
\end{array}\right) \rightarrow \mathcal{A}:\{a, b\} \mapsto \Lambda^{(2)}(a b), \\
& \Lambda^{(z)}:\left(\begin{array}{c}
X \\
\leq 2
\end{array}\right) \rightarrow \mathcal{A}:\{a, b\} \mapsto \Lambda^{(z)}(a b), \\
& \Lambda^{(3)}:\left(\begin{array}{c}
X \\
\leq 3
\end{array}\right) \rightarrow \mathcal{A}:\{a, b, c\} \mapsto \Lambda^{(3)}(a b c) .
\end{aligned}
$$


Regarding these definitions, we will establish the following facts:

Proposition 1.1. Continuing with the definitions and notations introduced above, one has

$$
\Sigma^{(2)} \in \mathcal{P}_{(2)}(X \mid \mathcal{A}), \quad \Sigma^{(z)} \in \mathcal{P}_{\leq 2}(X, z \mid \mathcal{A}), \quad \Sigma^{(3)} \in \mathcal{P}_{(3)}(X \mid \mathcal{A}),
$$

and

$$
\Sigma^{(\mathrm{bil})} \in \mathcal{L}_{2}(X \mid \mathcal{A})
$$

for every map $\Sigma \in \mathcal{S}^{*}(X \mid \mathcal{A})$ implying that, associating to any $\Sigma \in \mathcal{S}^{*}(X \mid \mathcal{A})$ the maps $\Sigma^{(2)}, \Sigma^{(z)}, \Sigma^{(3)}$, and $\Sigma^{(\mathrm{bil})}$, respectively, defines canonical group homomorphisms

$$
\begin{aligned}
\sigma_{2}: \mathcal{S}^{*}(X \mid \mathcal{A}) & \rightarrow \mathcal{P}_{(2)}(X \mid \mathcal{A}): \\
\Sigma & \mapsto\left(\Sigma^{(2)}:\{a, b\} \mapsto \Sigma_{+}(a \mid b)\right) \\
\sigma_{z}: \mathcal{S}^{*}(X \mid \mathcal{A}) & \rightarrow \mathcal{P}_{\leq 2}(X, z \mid \mathcal{A}): \\
\Sigma & \mapsto\left(\Sigma^{(z)}:\{a, b\} \mapsto \Sigma_{+}(a b \mid z)\right), \\
\sigma_{3}: \mathcal{S}^{*}(X \mid \mathcal{A}) & \rightarrow \mathcal{P}_{(3)}(X \mid \mathcal{A}): \\
\Sigma & \mapsto\left(\Sigma^{(3)}:\{a, b, c\} \mapsto \Sigma_{+}(a b \mid c)+\Sigma_{+}(b c \mid a)+\Sigma_{+}(c a \mid b)\right),
\end{aligned}
$$

and

$$
\begin{aligned}
\sigma_{\mathrm{bil}}: \mathcal{S}^{*}(X \mid \mathcal{A}) & \rightarrow \mathcal{L}_{2}(X \mid \mathcal{A}): \\
\Sigma & \mapsto\left(\Sigma^{(\mathrm{bil})}:((x, y),(u, v)) \mapsto \Sigma_{+}(x v \mid y u)-\Sigma_{+}(x u \mid y v)\right)
\end{aligned}
$$

which group homomorphisms will also be called "trace homomorphisms" associated with the set $X$.

Furthermore, the trace homomorphisms $\sigma_{z}, \sigma_{3}$, and $\sigma_{\text {bil }}$ are split-surjective in case $X$ is a finite set, i.e., there exist group homomorphisms $\zeta_{s}, \psi_{s}$, and $\lambda_{s}$ back from $\mathcal{P}_{\leq 2}(X, z \mid \mathcal{A}), \mathcal{P}_{(3)}(X \mid \mathcal{A})$, and $\mathcal{L}_{2}(X \mid \mathcal{A})$, respectively, into $\mathcal{S}^{*}(X \mid \mathcal{A})$ such that

$$
\sigma_{z} \circ \zeta_{s}=\operatorname{Id}_{\mathcal{P}_{\leq 2}(X, z \mid \mathcal{A})}, \quad \sigma_{3} \circ \psi_{s}=\operatorname{Id}_{\mathcal{P}_{(3)}(X \mid \mathcal{A})}, \quad \text { and } \quad \sigma_{\text {bil }} \circ \lambda_{s}=\operatorname{Id}_{\mathcal{L}_{2}(X \mid \mathcal{A})}
$$

holds, and the same holds for the trace homomorphisms $\sigma_{2}$ in that case provided that $\mathcal{A}$ does not contain any element of order 2.

Proposition 1.2. Further continuing with the definitions and notations introduced above, one has

$$
\Pi^{(2)} \in \mathcal{P}_{(2)}(X \mid \mathcal{A}), \quad \Pi^{(z)} \in \mathcal{P}_{\leq 2}(X, z \mid \mathcal{A}), \quad \Pi^{(3)} \in \mathcal{P}_{(3)}(X \mid \mathcal{A})
$$

and

$$
\Pi^{(\text {bil })} \in \mathcal{L}_{2}(X \mid \mathcal{A})
$$


for every map $\Pi \in \mathcal{P}_{\leq 2}(X \mid \mathcal{A})$ implying that, associating to any map $\Pi$ in $\mathcal{P}_{\leq 2}(X \mid \mathcal{A})$ the maps

$$
\Pi^{(2)} \in \mathcal{P}_{(2)}(X \mid \mathcal{A}), \quad \Pi^{(z)} \in \mathcal{P}_{\leq 2}(X, z \mid \mathcal{A}), \quad \Pi^{(3)} \in \mathcal{P}_{(3)}(X \mid \mathcal{A})
$$

and

$$
\Pi^{(\text {bil })} \in \mathcal{L}_{2}(X \mid \mathcal{A}),
$$

respectively, defines canonical group homomorphisms

$$
\begin{aligned}
& \omega_{2}: \mathcal{P}_{\leq 2}(X \mid \mathcal{A}) \rightarrow \mathcal{P}_{(2)}(X \mid \mathcal{A}): \\
& \Pi \mapsto\left(\Pi^{(2)}:\{a, b\} \mapsto \Pi^{(2)}(a b)\right), \\
& \omega_{z}: \mathcal{P}_{\leq 2}(X \mid \mathcal{A}) \rightarrow \mathcal{P}_{\leq 2}(X, z \mid \mathcal{A}): \\
& \Pi \mapsto\left(\Pi^{(z)}:\{a, b\} \mapsto \Pi^{(z)}(a b)\right), \\
& \omega_{3}: \mathcal{P}_{\leq 2}(X \mid \mathcal{A}) \rightarrow \mathcal{P}_{(3)}(X \mid \mathcal{A}): \\
& \Pi \mapsto\left(\Pi^{(3)}:\{a, b, c\} \mapsto \Pi^{(3)}(a b c)\right),
\end{aligned}
$$

and

$$
\begin{aligned}
\omega_{\text {bil }}: \mathscr{P}_{\leq 2}(X \mid \mathcal{A}) & \rightarrow \mathcal{L}_{2}(X \mid \mathcal{A}): \\
\Pi & \mapsto\left(\Pi^{(\mathrm{bil})}:((x, y),(u, v)) \mapsto \Pi^{(\mathrm{bil})}(x y: u v)\right) .
\end{aligned}
$$

Furthermore, one has

$$
\left(\Pi^{(2)}\right)^{(z)}=-2 \Pi^{(z)} \text { and }\left(\Pi^{(z)}\right)^{(2)}=\Pi^{(2)}
$$

for every $\Pi \in \mathcal{P}_{\leq 2}(X \mid \mathcal{A})$ and, therefore, in view of the fact that $\Pi^{(z)}=\Pi$ holds for every $\Pi \in \mathcal{P}_{\leq 2}(X, z \mid \mathcal{A})$, and $\Pi^{(2)}=-2 \Pi$ for every $\Pi \in \mathcal{P}_{(2)}(X \mid \mathcal{A})$, also

$$
\left(\Pi^{(2)}\right)^{(z)}=-2 \Pi
$$

for every $\Pi \in \mathcal{P}_{\leq 2}(X, z \mid \mathcal{A})$, and

$$
\left(\Pi^{(z)}\right)^{(2)}=-2 \Pi
$$

for every $\Pi \in \mathcal{P}_{(2)}(X \mid \mathcal{A})$. In addition, given any map $\Pi \in \mathcal{P}_{(2)}(X \mid \mathcal{A})$, one can find some map $\Pi_{z} \in \mathcal{P}_{\leq 2}(X, z \mid \mathcal{A})$ for which $\omega_{2}\left(\Pi_{z}\right)=\Pi$ holds.

And finally, one also has

$$
\omega_{2}\left(\sigma_{z}(\Sigma)\right)=\sigma_{2}(\Sigma), \quad \omega_{3}\left(\sigma_{z}(\Sigma)\right)=\sigma_{3}(\Sigma), \quad \omega_{\text {bil }}\left(\sigma_{z}(\Sigma)\right)=\sigma_{\text {bil }}(\Sigma)
$$

and

$$
\omega_{z}\left(\sigma_{2}(\Sigma)\right)=-2 \sigma_{z}(\Sigma), \quad \omega_{3}\left(\sigma_{2}(\Sigma)\right)=-2 \sigma_{3}(\Sigma), \quad \omega_{\text {bil }}\left(\sigma_{2}(\Sigma)\right)=-2 \sigma_{\text {bil }}(\Sigma),
$$

for every $\Sigma \in \mathcal{S}^{*}(X \mid \mathcal{A})$. 
In other words, denoting

(i) the restrictions $\left.\omega_{z}\right|_{\mathcal{P}_{(2)}(X \mid \mathcal{A})},\left.\omega_{3}\right|_{\mathcal{P}_{(2)}(X \mid \mathcal{A})}$, and $\left.\omega_{\text {bil }}\right|_{\mathcal{P}_{(2)}(X \mid \mathcal{A})}$ of the group homomorphisms of $\omega_{z}, \omega_{3}$, and $\omega_{\text {bil }}$ to the subgroup $\mathcal{P}_{(2)}(X \mid \mathcal{A})$ of $\mathcal{P}_{\leq 2}(X \mid \mathcal{A})$ by $\pi_{z}$, $\pi_{3}$, and $\pi_{\mathrm{bil}}$, respectively,

(ii) and the restrictions $\left.\omega_{2}\right|_{\mathcal{P}_{\leq 2}(X, z \mid \mathcal{A})},\left.\omega_{3}\right|_{\mathcal{P}_{\leq 2}(X, z \mid \mathcal{A})}$, and $\left.\omega_{\text {bil }}\right|_{\mathcal{P}_{\leq 2}(X, z \mid \mathcal{A})}$ of the group homomorphisms $\omega_{2}, \omega_{3}$, and $\omega_{\text {bil }}$ to the subgroup $\mathcal{P}_{\leq 2}(X, z \mid \mathcal{A})$ of $\mathcal{P}_{\leq 2}(X \mid \mathcal{A})$ by $\zeta_{2}, \zeta_{3}$, and $\zeta_{\text {bil }}$, respectively,

one obtains a surjective group homomorphism $\zeta_{2}: \mathcal{P}_{\leq 2}(X, z \mid \mathcal{A}) \rightarrow \mathcal{P}_{(2)}(X \mid \mathcal{A})$, one has

$$
\pi_{z} \circ \zeta_{2}=-2 \operatorname{Id}_{\mathcal{P}_{\leq 2}(X, z \mid \mathcal{A})}, \quad \zeta_{2} \circ \pi_{z}=-2 \operatorname{Id}_{\mathcal{P}_{(2)}(X \mid \mathcal{A})}
$$

and

$$
\zeta_{2} \circ \sigma_{z}=\sigma_{2}, \quad \zeta_{3} \circ \sigma_{z}=\sigma_{3}, \quad \zeta_{\text {bil }} \circ \sigma_{z}=\sigma_{\text {bil }},
$$

as well as

$$
\pi_{z} \circ \sigma_{2}=-2 \sigma_{z}, \quad \pi_{3} \circ \sigma_{2}=-2 \sigma_{3}, \quad \pi_{\mathrm{bil}} \circ \sigma_{2}=-2 \sigma_{\mathrm{bil}},
$$

that is, one obtains the following diagram

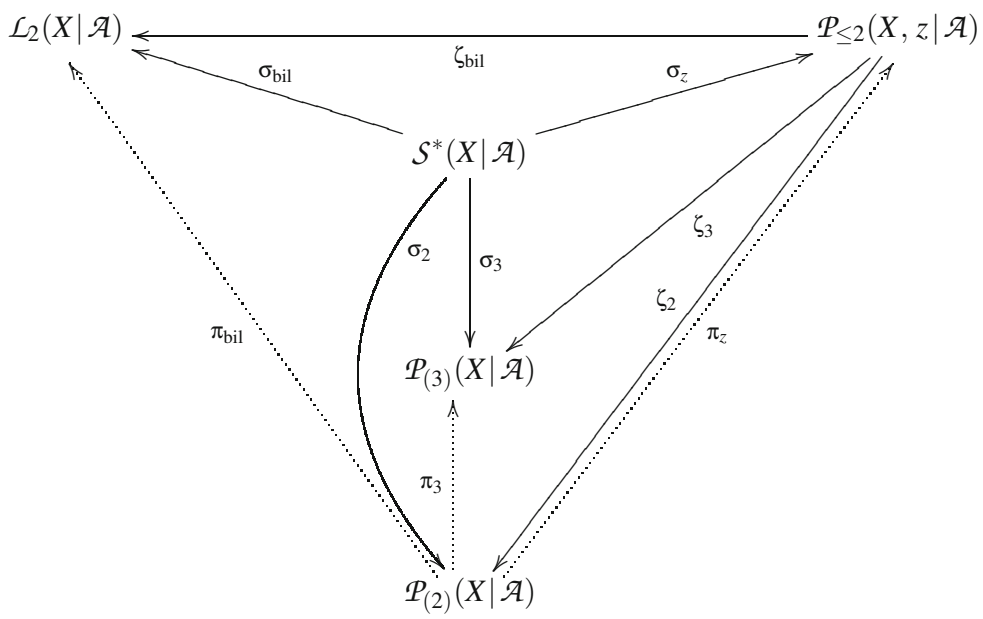

in which

(i) the trace homomorphisms $\sigma_{\text {bil }}, \sigma_{z}$, and $\sigma_{3}$ emanating from the group $\mathcal{S}^{*}(X \mid \mathcal{A})$ in its center are split-surjective in case $X$ is a finite set, and the same holds for the trace homomorphisms $\sigma_{2}$ in that case provided that $\mathcal{A}$ does not contain any element of order 2 ,

(ii) the group homomorphism $\zeta_{2}: \mathcal{P}_{\leq 2}(X, z \mid \mathcal{A}) \rightarrow \mathcal{P}_{(2)}(X \mid \mathcal{A})$ is surjective,

(iii) and all triangles commute except those five involving exactly one dotted arrow (including the two "degenerate" triangles one obtains by adding, to the two anti-parallel arrows connecting the groups $\mathcal{P}_{(2)}(X \mid \mathcal{A})$ and $\mathcal{P}_{\leq 2}(X, z \mid \mathcal{A})$, 
the identity homomorphism of either of these two groups) while composing, in any one of those five triangles, the homomorphism represented by its dotted arrow with the homomorphisms represented by its straight arrow directed towards the group $\mathcal{P}_{(2)}(X \mid \mathcal{A})$ always yields -2 times the homomorphisms represented by its other straight arrow (expressing, in terms of the diagram under consideration, the fact that $\pi_{z} \circ \sigma_{2}=-2 \sigma_{z}, \pi_{3} \circ \sigma_{2}=-2 \sigma_{3}, \quad \pi_{\text {bil }} \circ \sigma_{2}=-2 \sigma_{\text {bil }}$, $\pi_{z} \circ \zeta_{2}=-2 \operatorname{Id}_{\mathcal{P}_{\leq 2}(X, z \mid \mathcal{A})}$, and $\zeta_{2} \circ \pi_{z}=-2 \operatorname{Id}_{\mathcal{P}_{(2)}(X \mid \mathcal{A})}$ holds $)$.

Proposition 1.3. Further continuing with the definitions and notations introduced above, one has

$$
\Psi^{(2)} \in \mathcal{P}_{(2)}(X \mid \mathcal{A}), \quad \Psi^{(z)} \in \mathcal{P}_{\leq 2}(X, z \mid \mathcal{A}), \quad \text { and } \Psi^{(\mathrm{bil})} \in \mathcal{L}_{2}(X \mid \mathcal{A})
$$

for every map $\Psi \in \mathcal{P}_{(3)}(X \mid \mathcal{A})$, implying that, associating to any map $\Psi$ in $\mathcal{P}_{(3)}(X \mid \mathcal{A})$, the maps

$$
\Psi^{(2)} \in \mathcal{P}_{(2)}(X \mid \mathcal{A}), \quad \Psi^{(z)} \in \mathcal{P}_{\leq 2}(X, z \mid \mathcal{A}), \quad \text { and } \Psi^{(\mathrm{bil})} \in \mathcal{L}_{2}(X \mid \mathcal{A}),
$$

respectively, defines canonical group homomorphisms

$$
\begin{aligned}
\Psi_{2}: \mathcal{P}_{(3)}(X \mid \mathcal{A}) & \rightarrow \mathcal{P}_{(2)}(X \mid \mathcal{A}): \\
\Psi & \mapsto\left(\Psi^{(2)}:\{a, b\} \mapsto \Psi(a b)\right), \\
\Psi_{z}: \mathcal{P}_{(3)}(X \mid \mathcal{A}) & \rightarrow \mathcal{P}_{\leq 2}(X, z \mid \mathcal{A}): \\
\Psi & \mapsto\left(\Psi^{(z)}:\{a, b\} \mapsto \Psi(a b z)-\Psi(a b\}\right),
\end{aligned}
$$

and

$$
\begin{aligned}
\psi_{\text {bil }}: \mathcal{P}_{(3)}(X \mid \mathcal{A}) & \rightarrow \mathcal{L}_{2}(X \mid \mathcal{A}): \\
\Psi & \mapsto\left(\Psi^{(\mathrm{bil})}:((x, y),(u, v)) \mapsto \Psi^{(\mathrm{bil})}(x y: u v)\right)
\end{aligned}
$$

(where, remarkably, $\Psi^{(\mathrm{bil})}(x y: u v)$, being defined as $\Psi(x y v)-\Psi(x v)-\Psi(x y u)+$ $\Psi(x u)$ also coincides with the term $\Psi(x y u)+\Psi(y v)-\Psi(x y v)-\Psi(y u)$ for every $\Psi \in$ $\left.\mathcal{P}_{(3)}(X \mid \mathcal{A})\right)$ for which the identities

$$
\begin{array}{lll}
\psi_{2} \circ \sigma_{3}=\sigma_{2}, & \psi_{z} \circ \sigma_{3}=\sigma_{z}, & \psi_{\text {bil }} \circ \sigma_{3}=\sigma_{\text {bil }}, \\
\psi_{2} \circ \pi_{3}=-2 \operatorname{Id}_{\mathcal{P}_{(2)}(X \mid \mathcal{A})}, & \psi_{z} \circ \pi_{3}=\pi_{z}, & \psi_{\text {bil }} \circ \pi_{3}=\pi_{\text {bil }}, \\
\psi_{2} \circ \zeta_{3}=\zeta_{2}, & \psi_{z} \circ \zeta_{3}=\operatorname{Id}_{\mathcal{P}_{\leq 2}(X, z \mid \mathcal{A}),} & \psi_{\text {bil }} \circ \zeta_{3}=\zeta_{\text {bil }}, \\
\pi_{z} \circ \psi_{2}=-2 \psi_{z}, & \pi_{3} \circ \psi_{2}=-2 \operatorname{Id}_{\mathcal{P}_{(3)}(X \mid \mathcal{A}),} & \pi_{\text {bil }} \circ \psi_{2}=-2 \psi_{\text {bil }},
\end{array}
$$

and

$$
\zeta_{2} \circ \psi_{z}=\psi_{2}, \quad \zeta_{3} \circ \psi_{z}=\operatorname{Id}_{\mathcal{P}_{(3)}(X \mid \mathcal{A})}, \quad \zeta_{\text {bil }} \circ \psi_{z}=\psi_{\text {bil }},
$$

hold. 
In other words, our diagram above can be extended so as to yield the diagram

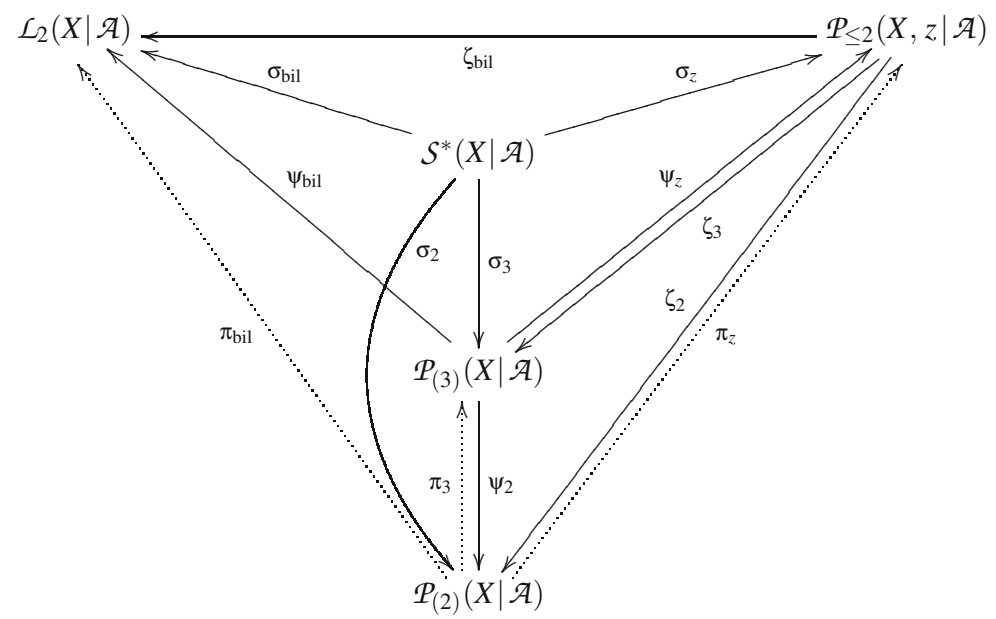

in which now all triangles commute except those eleven triangles involving exactly one dotted arrow (including the four degenerate triangles one obtains by adding, to the two pairs of anti-parallel arrows involving one dotted arrow, the identity homomorphism of either of the two groups connected by them) while composing, in any one of those eleven triangles, the homomorphism represented by its dotted arrow with the homomorphism represented by its straight arrow directed towards the group $\mathcal{P}_{(2)}(X \mid \mathcal{A})$ always yields as before -2 times the homomorphisms represented by its other straight arrow.

Proposition 1.4. Continuing further with the definitions and notations introduced above, one has

$$
\Lambda^{(2)} \in \mathcal{P}_{(2)}(X \mid \mathcal{A}), \quad \Lambda^{(z)} \in \mathcal{P}_{\leq 2}(X, z \mid \mathcal{A}), \quad \text { and } \Lambda^{(3)} \in \mathcal{P}_{(3)}(X \mid \mathcal{A})
$$

for every map $\Lambda \in \mathcal{L}_{2}(X \mid \mathcal{A})$, implying that, associating to any map $\Lambda$ in $\mathcal{P}_{(3)}(X \mid \mathcal{A})$, the maps $\Lambda^{(2)}, \Lambda^{(z)}$, and $\Lambda^{(3)}$, define canonical group homomorphisms

$$
\begin{aligned}
\lambda_{2}: \mathcal{L}_{2}(X \mid \mathcal{A}) & \rightarrow \mathcal{P}_{(2)}(X \mid \mathcal{A}): \\
\Lambda & \mapsto\left(\Lambda^{(2)}:\{a, b\} \mapsto \Lambda(a b: b a)\right), \\
\lambda_{z}: \mathcal{L}_{2}(X \mid \mathcal{A}) & \rightarrow \mathcal{P}_{\leq 2}(X, z \mid \mathcal{A}): \\
\Lambda & \mapsto\left(\Lambda^{(z)}:\{a, b\} \mapsto \Lambda(a z: z b)\right),
\end{aligned}
$$

and

$$
\begin{aligned}
\lambda_{3}: \mathcal{L}_{2}(X \mid \mathcal{A}) & \rightarrow \mathcal{P}_{(3)}(X \mid \mathcal{A}): \\
\Lambda & \mapsto\left(\Lambda^{(3)}:\{a, b, c\} \mapsto \Lambda^{(3)}(a b c)\right)
\end{aligned}
$$


for which the identities

$$
\begin{array}{lll}
\lambda_{2} \circ \sigma_{\mathrm{bil}}=\sigma_{2}, & \lambda_{z} \circ \sigma_{\mathrm{bil}}=\sigma_{z}, & \lambda_{3} \circ \sigma_{\mathrm{bil}}=\sigma_{3}, \\
\lambda_{2} \circ \pi_{\mathrm{bil}}=-2 \operatorname{Id}_{\mathcal{P}_{(2)}(X \mid \mathcal{A})}, & \lambda_{z} \circ \pi_{\mathrm{bil}}=\pi_{z}, & \lambda_{3} \circ \pi_{\mathrm{bil}}=\pi_{3}, \\
\lambda_{2} \circ \zeta_{\mathrm{bil}}=\zeta_{2}, & \lambda_{z} \circ \zeta_{\mathrm{bil}}=\operatorname{Id}_{\mathcal{P}_{\leq 2}(X, z \mid \mathcal{A})}, & \lambda_{3} \circ \zeta_{\mathrm{bil}}=\zeta_{3}, \\
\lambda_{2} \circ \psi_{\mathrm{bil}}=\psi_{2}, & \lambda_{z} \circ \psi_{\mathrm{bil}}=\psi_{z}, & \lambda_{3} \circ \psi_{\mathrm{bil}}=\operatorname{Id}_{\mathcal{P}_{(3)}(X \mid \mathcal{A}),}, \\
\pi_{z} \circ \lambda_{2}=-2 \lambda_{z}, & \pi_{3} \circ \lambda_{2}=-2 \lambda_{3}, & \pi_{\mathrm{bil}} \circ \lambda_{2}=-2 \operatorname{Id}_{\mathcal{L}_{2}(X \mid \mathcal{A})}, \\
\zeta_{2} \circ \lambda_{z}=\lambda_{2}, & \zeta_{3} \circ \lambda_{z}=\operatorname{Id}_{\mathcal{L}_{2}(X \mid \mathcal{A})}, & \zeta_{\mathrm{bil}} \circ \lambda_{z}=\lambda_{3},
\end{array}
$$

and

$$
\psi_{2} \circ \lambda_{3}=\lambda_{2}, \quad \psi_{z} \circ \lambda_{3}=\lambda_{z}, \quad \psi_{\text {bil }} \circ \lambda_{3}=\operatorname{Id}_{\mathcal{L}_{2}(X \mid \mathcal{A})}
$$

hold.

Thus, our results allow us to extend our diagram even further, giving altogether, rise to the following theorem:

Theorem 1.5. Given any set $X$ and any abelian group $\mathcal{A}$, the groups and group homomorphisms introduced above all fit into one big diagram

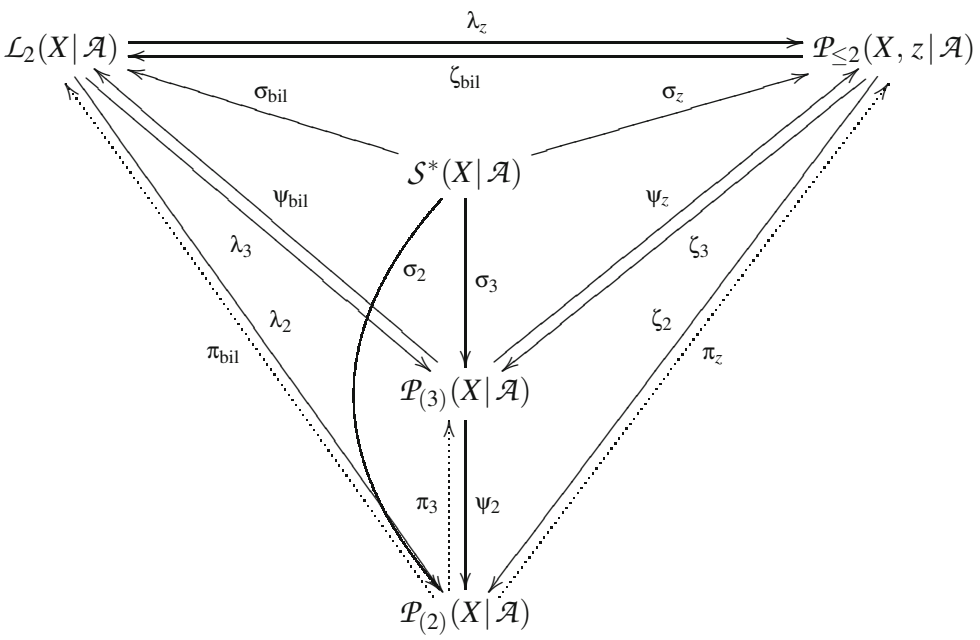

in which

(i) the trace homomorphisms $\sigma_{\mathrm{bil}}, \sigma_{z}$, and $\sigma_{3}$ represented by arrows emanating from the group $S^{*}(X \mid \mathcal{A})$ in its center are split-surjective in case $X$ is finite, and the same holds for the trace homomorphisms $\sigma_{2}$ in that case provided $\mathcal{A}$ does not contain any element of order 2 , 
(ii) the homomorphisms $\zeta_{2}, \psi_{2}$, and $\lambda_{2}$ represented by arrows directed towards the group $\mathcal{P}_{(2)}(X \mid \mathcal{A})$ are all surjective,

(iii) all pairs of anti-parallel solid arrows represent mutually inverse group homomorphisms,

(iv) all triangles commute except those fifteen triangles involving exactly one dotted arrow, including the six degenerate triangles one obtains by inserting, in between any one of the three pairs of anti-parallel arrows involving one dotted arrow, the identity homomorphism of either of the two groups connected by them,

(iv) and composing the homomorphisms represented by the dotted arrow in any one of those fifteen triangles involving exactly one dotted arrow with the homomorphism represented by the arrow directed towards the group $\mathcal{P}_{(2)}(X \mid \mathcal{A})$ always yields -2 times the homomorphism represented by its third arrow.

In the next section, we will collect some useful observations. Then, we will establish Theorem 1.5. And in the last section, we will discuss the special situation arising in case $\mathcal{A}$ has no 2-torsion.

In the second and the third parts of this series of papers (cf. $[13,14])$, the main results of split-decomposition theory (cf. [4]) will be established within the conceptual framework developed here.

\section{Some Useful Observations}

The following simple observations will be useful:

Observation 2.1. Given any $\Sigma \in \mathcal{S}^{*}(X \mid \mathcal{A})$ and any two subsets $Y=\left\{y_{1}, \ldots, y_{i}\right\}$ and $Z=\left\{z_{1}, \ldots, z_{j}\right\}$ of $X$,

$$
\Sigma_{+}(Y \mid Z)=\Sigma_{+}(Y \cup\{x\} \mid Z)+\Sigma_{+}(Y \mid Z \cup\{x\}),
$$

or, equivalently,

$$
\Sigma_{+}\left(y_{1} \cdots y_{i} \mid z_{1} \cdots z_{j}\right)=\Sigma_{+}\left(y_{1} \cdots y_{i} x \mid z_{1} \cdots z_{j}\right)+\Sigma_{+}\left(y_{1} \cdots y_{i} \mid z_{1} \cdots z_{j} x\right)
$$

holds for any element $x \in X$.

Indeed, any split that separates $Y$ and $Z$ exclusively either separates $Y \cup\{x\}$ and $Z$, or separates $Y$ and $Z \cup\{x\}$.

Observation 2.2. Given any map $\Sigma \in \mathcal{S}^{*}(X \mid \mathcal{A})$ and any two subsets $Y=\left\{y_{1}, \ldots, y_{i}\right\}$ and $Z=\left\{z_{1}, \ldots, z_{j}\right\}$ of $X$,

$$
\Sigma_{+}(Y \cup\{v\} \mid Z)-\Sigma_{+}(Y \cup\{u\} \mid Z)=\Sigma_{+}(Y \cup\{v\} \mid Z \cup\{u\})-\Sigma_{+}(Y \cup\{u\} \mid Z \cup\{v\}),
$$

or, equivalently,

$$
\begin{aligned}
\Sigma_{+}\left(y_{1} \cdots y_{i} v \mid z_{1} \cdots z_{j}\right)-\Sigma_{+}\left(y_{1} \cdots y_{i} u \mid z_{1} \cdots z_{j}\right)= & \Sigma_{+}\left(y_{1} \cdots y_{i} v \mid z_{1} \cdots z_{j} u\right) \\
& -\Sigma_{+}\left(y_{1} \cdots y_{i} u \mid z_{1} \cdots z_{j} v\right)
\end{aligned}
$$

holds for any two elements $u, v \in X$. 
Indeed, Observation 2.1 implies that also

$$
\begin{aligned}
\Sigma_{+}(Y \cup\{v\} \mid Z)-\Sigma_{+}(Y \cup\{u\} \mid Z)= & \Sigma_{+}(Y \cup\{u, v\} \mid Z)+\Sigma_{+}(Y \cup\{v\} \mid Z \cup\{u\}) \\
& -\Sigma_{+}(Y \cup\{u, v\} \mid Z)-\Sigma_{+}(Y \cup\{u\} \mid Z \cup\{v\}) \\
= & \Sigma_{+}(Y \cup\{v\} \mid Z \cup\{u\})-\Sigma_{+}(Y \cup\{u\} \mid Z \cup\{v\})
\end{aligned}
$$

holds for every map $\Sigma \in \mathcal{S}^{*}(X \mid \mathcal{A})$, any two subsets $Y$ and $Z$ of $X$, and any two elements $u, v \in X$.

Observation 2.3. Given any map $\Psi \in \mathcal{P}_{\leq 3}(X \mid \mathcal{A})$, the following three assertions are equivalent:

(i) $\Psi$ is an element of the subgroup $\mathcal{P}_{(3)}(X \mid \mathcal{A})$ of $\mathcal{P}_{\leq 3}(X \mid \mathcal{A})$,

(ii) one has $\sum_{I \in\left(\begin{array}{c}\{1,2,3,4\} \\ 3\end{array}\right)} \Psi\left(\left\{a_{i}: i \in I\right\}\right)=\sum_{J \in(\underset{2}{\{1,2,3,4\}})} \Psi\left(\left\{a_{j}: j \in J\right\}\right)$ for all $a_{1}, a_{2}$ : $a_{3}, a_{4} \in X$ and

$$
2 \Psi(a b c)=\Psi(a b)+\Psi(b c)+\Psi(c a)
$$

for all $a, b, c \in X$,

(iii) one has

$$
2 \Psi(a b c)=\Psi(a b)+\Psi(b c)+\Psi(c a)
$$

and

$$
\Psi(a b d)+\Psi(b c d)+\Psi(c a d)=\Psi(a b c)+\Psi(a d)+\Psi(b d)+\Psi(c d)
$$

for all $a, b, c, d \in X$.

Indeed, putting $a_{1}:=a, a_{2}:=b$, and $a_{3}=a_{4}:=c$ in the identity

$$
\sum_{I \in\left(\begin{array}{c}
\{1,2,3,4\} \\
3
\end{array}\right)} \Psi\left(\left\{a_{i}: i \in I\right\}\right)=\sum_{J \in\left(\begin{array}{c}
\{1,2,3,4\} \\
2
\end{array}\right)} \Psi\left(\left\{a_{j}: j \in J\right\}\right),
$$

one gets

$$
2 \Psi(a b c)+\Psi(a c)+\Psi(b c)=\Psi(a b)+\Psi(b c)+\Psi(c a)+\Psi(a c)+\Psi(b c)+\Psi(c),
$$

and, therefore,

$$
2 \Psi(a b c)=\Psi(a b)+\Psi(b c)+\Psi(c a)
$$

in case $\Psi(c)=0$ holds, showing that (i) implies (ii). Conversely, putting $b:=c:=a$ in the last identity, one gets $2 \Psi(a)=3 \Psi(a)$ and, therefore, $\Psi(a)=0$, showing that (ii) also implies (i). Furthermore, if $2 \Psi(a b c)=\Psi(a b)+\Psi(b c)+\Psi(c a)$ holds for all $a, b, c \in X$, one has

$$
\begin{aligned}
\Psi(a b c)+\Psi(b c d)+\Psi(c d a)+\Psi(d a b)= & \Psi(a b)+\Psi(b c)+\Psi(c a)+\Psi(a d) \\
& +\Psi(b d)+\Psi(c d)
\end{aligned}
$$

for some $a, b, c, d \in X$ if and only if one has

$$
\Psi(a b c)+\Psi(b c d)+\Psi(c d a)+\Psi(d a b)=\Psi(a b c)+\Psi(a d)+\Psi(b d)+\Psi(c d),
$$


if and only if one has

$$
\Psi(b c d)+\Psi(c d a)+\Psi(d a b)=\Psi(a b c)+\Psi(a d)+\Psi(b d)+\Psi(c d) .
$$

So, (ii) is also equivalent with (iii).

Observation 2.4. Given any map $\Psi \in \mathcal{P}_{(3)}(X \mid \mathcal{A})$, one has

$$
\begin{aligned}
\Psi^{(z)}(a b) & =\Psi(a b z)-\Psi(a b) \\
& =\Psi(a z)+\Psi(b z)-\Psi(a b z)
\end{aligned}
$$

for all elements $a, b, c \in X$.

Indeed, one has

$$
\begin{aligned}
\Psi^{(z)}(a b) & =\Psi(a b z)-\Psi(a b) \\
& =2 \Psi(a b z)-\Psi(a b)-\Psi(a b z) \\
& =\Psi(a b)+\Psi(a z)+\Psi(b z)-\Psi(a b)-\Psi(a b z) \\
& =\Psi(a z)+\Psi(b z)-\Psi(a b z)
\end{aligned}
$$

for every map $\Psi \in \mathcal{P}_{(3)}(X \mid \mathcal{A})$ and all elements $a, b, z \in X$.

Observation 2.5. Given any map $\Psi \in \mathcal{P}_{(3)}(X \mid \mathcal{A})$, one has

$$
\Psi^{(\mathrm{bil})}(x y: u v)=\Psi^{(y)}(x v)-\Psi^{(y)}(x u),
$$

for all elements $x, y, u, v \in X$.

Indeed, by definition, one has

$$
\Psi^{(\mathrm{bil})}(x y: u v)=\Psi(x y v)-\Psi(x v)-\Psi(x y u)-\Psi(x u)=\Psi^{(y)}(x v)-\Psi^{(y)}(x u)
$$

for all elements $x, y, u, v \in X$.

Observation 2.6. Given any map $\Lambda \in \mathcal{L}_{2}(X \mid \mathcal{A})$, one has

$$
\begin{gathered}
\Lambda(x x: u v)=\Lambda(x y: u u)=0 \\
\Lambda(x y: u v)+\Lambda(y x: u v)=\Lambda(x y: u v)+\Lambda(x y: v u)=0
\end{gathered}
$$

or, equivalently,

$$
\Lambda(y x: u v)=-\Lambda(x y: u v) \quad \text { and } \quad \Lambda(x y: v u)=-\Lambda(x y: u v)
$$

and

$$
\Lambda(x y: u v)=\Lambda(y x: v u)=\Lambda(u v: x y)=\Lambda(v u: y x)
$$

for all $x, y, u, v \in X$. 
Indeed, bilinearity implies that

$$
\Lambda(x x: u v)+\Lambda(x x: u v)=\Lambda(x x: u v)
$$

and, therefore, $\Lambda(x x: u v)=0$ holds for all $x, u, v \in X$. Thus, symmetry implies that also $\Lambda(x y: u u)=0$ holds for all $x, y, u \in X$, and bilinearity implies further that also $\Lambda(x y: u v)+\Lambda(y x: u v)=\Lambda(x x: u v)=0$ and $\Lambda(x y: u v)+\Lambda(x y: v u)=\Lambda(x y: u u)=0$ and, therefore, also

$$
\Lambda(x y: u v)=-\Lambda(y x: u v)=\Lambda(y x: v u)=\Lambda(v u: y x)
$$

holds for all $x, y, u, v \in X$.

Observation 2.7. Given any map $\Lambda \in \mathcal{L}_{2}(X \mid \mathcal{A})$, one has

$$
\Lambda(x y: u v)+\Lambda(x u: v y)+\Lambda(x v: y u)=0
$$

for all $x, y, u, v \in X$.

Indeed, one has

$$
\begin{aligned}
\Lambda(x y: u v)+\Lambda(x u: v y)+\Lambda(x v: y u)= & \Lambda(x y: u v)+\Lambda(x y: v y) \\
& +\Lambda(y u: v y)+\Lambda(x v: y u) \\
= & \Lambda(x y: u y)+\Lambda(v y: y u)+\Lambda(x v: y u) \\
= & \Lambda(x y: u y)+\Lambda(x y: y u) \\
= & \Lambda(x y: u u) \\
= & 0,
\end{aligned}
$$

for all $\Lambda \in \mathcal{L}_{2}(X \mid \mathcal{A})$ and $x, y, u, v \in X$.

Observation 2.8. Given any map $\Lambda \in \mathcal{L}_{2}(X \mid \mathcal{A})$, one has

$$
\begin{aligned}
\Lambda(x y: u v) & =\Lambda(x z: u v)+\Lambda(z y: u v) \\
& =\Lambda(x z: u z)+\Lambda(x z: z v)+\Lambda(z y: u z)+\Lambda(z y: z v) \\
& =\Lambda(x z: z v)+\Lambda(y z: z u)-\Lambda(x z: z u)-\Lambda(y z: z v)
\end{aligned}
$$

for all $x, y, u, v, z \in X$.

Observation 2.9. Given any map $\Lambda \in \mathcal{L}_{2}(X \mid \mathcal{A})$, one has

$$
\Lambda(x u: u x)+\Lambda(y v: v y)-\Lambda(x v: v x)-\Lambda(y u: u y)=2 \Lambda(x y: u v)
$$

for all $x, y, u, v \in X$. 
Indeed, one has

$$
\begin{aligned}
& \Lambda(x u: u x)+\Lambda(y v: v y)-\Lambda(x v: v x)-\Lambda(y u: u y) \\
& =(\Lambda(x u: u v)+\Lambda(x u: v x))+(\Lambda(y v: v u)+\Lambda(y v: u y))-\Lambda(x v: v x)-\Lambda(y u: u y) \\
& =\Lambda(x u: u v)+(\Lambda(v x: v x)+\Lambda(x u: v x))+\Lambda(y v: v u)+(\Lambda(u y: u y)+\Lambda(y v: u y)) \\
& =\Lambda(x u: u v)+\Lambda(v u: v x)+\Lambda(y v: v u)+\Lambda(u v: u y) \\
& =(\Lambda(x u: u v)+\Lambda(u y: u v))+(\Lambda(v u: y v)+\Lambda(v u: v x)) \\
& =\Lambda(x y: u v)+\Lambda(v u: y x) \\
& =2 \Lambda(x y: u v)
\end{aligned}
$$

for all $x, y, u, v \in X$.

\section{Proof of Theorem $\mathbf{1 . 5}$}

We can now start proving Theorem 1.5. We proceed in a step-by-step fashion, establishing the required facts from 3.1 to 3.20 below.

As before, we will also assume that a fixed element $z \in X$ is given.

Fact 3.1. Given any $\Pi \in \mathcal{P}_{\leq 2}(X \mid \mathcal{A})$, one has $\Pi^{(2)} \in \mathcal{P}_{(2)}(X \mid \mathcal{A})$ implying that, in particular, the group homomorphism

$$
\omega_{2}: \mathcal{P}_{\leq 2}(X \mid \mathcal{A}) \rightarrow \mathcal{P}_{(2)}(X \mid \mathcal{A}): \Pi \mapsto \Pi^{(2)}
$$

and its restriction

$$
\zeta_{2}: \mathcal{P}_{\leq 2}(X, z \mid \mathcal{A}) \rightarrow \mathcal{P}_{(2)}(X \mid \mathcal{A}): \Pi \mapsto \Pi^{(2)}
$$

are well defined.

Indeed, one has $\Pi^{(2)}(a)=\Pi(a)+\Pi(a)-2 \Pi(a)=0$ and

$$
\Pi^{(2)}(a b)+\Pi^{(2)}(b c)+\Pi^{(2)}(c a) \in 2 \mathcal{A}
$$

for all $a, b, c \in X$ in view of the fact that

$\Pi^{(2)}(a b)+\Pi^{(2)}(b c)+\Pi^{(2)}(c a)=2(\Pi(a)+\Pi(b)+\Pi(c)-\Pi(a b)-\Pi(b c)-\Pi(c a))$

holds for all $a, b, c \in X$.

Fact 3.2. Given any $\Pi \in \mathcal{P}_{\leq 2}(X \mid \mathcal{A})$, one has $\Pi^{(z)} \in \mathcal{P}_{\leq 2}(X, z \mid \mathcal{A})$ implying that, in particular, the group homomorphism

$$
\omega_{z}: \mathcal{P}_{\leq 2}(X \mid \mathcal{A}) \rightarrow \mathcal{P}_{\leq 2}(X, z \mid \mathcal{A}): \Pi \mapsto \Pi^{(z)}
$$

and its restriction

$$
\pi_{z}: \mathcal{P}_{(2)}(X \mid \mathcal{A}) \rightarrow \mathcal{P}_{\leq 2}(X, z \mid \mathcal{A}): \Pi \mapsto \Pi^{(z)}
$$

are well defined. 
Indeed, also $\Pi^{(z)}(a z)=\Pi(a z)+\Pi(z)-\Pi(a z)-\Pi(z)=0$ holds for every $a \in X$.

Fact 3.3. Given any $\Pi \in \mathcal{P}_{(2)}(X \mid \mathcal{A})$, one has $\Pi^{(2)}=-2 \Pi$.

This is obvious.

Fact 3.4. Given any $\Pi \in \mathcal{P}_{<2}(X, z \mid \mathcal{A})$, one has $\Pi^{(z)}=\Pi$.

This is also obvious.

Fact 3.5. Given any $\Pi \in \mathcal{P}_{\leq 2}(X \mid \mathcal{A})$, one has $\left(\Pi^{(z)}\right)^{(2)}=\Pi^{(2)}$.

Indeed, given any two elements $a, b \in X$, one has

$$
\begin{aligned}
\left(\Pi^{(z)}\right)^{(2)}(a b)= & \Pi^{(z)}(a)+\Pi^{(z)}(b)-2 \Pi^{(z)}(a b) \\
= & (\Pi(a)+\Pi(z)-2 \Pi(a z))+(\Pi(b)+\Pi(z)-2 \Pi(b z)) \\
& -2(\Pi(a b)+\Pi(z)-\Pi(a z)-\Pi(b z)) \\
= & \Pi(a)+\Pi(b)-2 \Pi(a b) \\
= & \Pi^{(2)}(a b),
\end{aligned}
$$

as claimed.

Fact 3.6. Given any $\Pi \in \mathcal{P}_{\leq 2}(X \mid \mathcal{A})$, one has $\left(\Pi^{(2)}\right)^{(z)}=-2 \Pi^{(z)}$.

Indeed, given any two elements $a, b \in X$, one has

$$
\begin{aligned}
\left(\Pi^{(2)}\right)^{(z)}(a b)= & \Pi^{(2)}(a b)+\Pi^{(2)}(z)-\Pi^{(2)}(a z)-\Pi^{(2)}(b z) \\
= & \Pi^{(2)}(a b)-\Pi^{(2)}(a z)-\Pi^{(2)}(b z) \\
= & (\Pi(a)+\Pi(b)-2 \Pi(a b))-(\Pi(a)+\Pi(z)-2 \Pi(a z)) \\
& -(\Pi(b)+\Pi(z)-2 \Pi(b z)) \\
= & -2 \Pi(a b)-2 \Pi(z)+2 \Pi(a z)+2 \Pi(b z) \\
= & -2 \Pi^{(z)}(a b),
\end{aligned}
$$

as claimed.

Fact 3.7. In particular, one has

$$
\pi_{z} \circ \zeta_{2}=-2 \operatorname{Id}_{\mathcal{P}_{\leq 2}(X, z \mid \mathcal{A})}
$$

and

$$
\zeta_{2} \circ \pi_{z}=-2 \operatorname{Id}_{\mathcal{P}_{(2)}(X \mid \mathcal{A})}
$$


Indeed, given any $\Pi \in \mathcal{P}_{\leq 2}(X, z \mid \mathcal{A})$, one has

$$
\left(\pi_{z} \circ \zeta_{2}\right)(\Pi)=\left(\Pi^{(2)}\right)^{(z)}=-2 \Pi^{(z)}=-2 \Pi
$$

and, given any $\Pi \in \mathcal{P}_{(2)}(X \mid \mathcal{A})$, one has

$$
\left(\zeta_{2} \circ \pi_{z}\right)(\Pi)=\left(\Pi^{(z)}\right)^{(2)}=\Pi^{(2)}=-2 \Pi,
$$

as claimed.

Fact 3.8. The group homomorphism $\zeta_{2}$ is surjective.

Indeed, given any map $\Pi \in \mathcal{P}_{(2)}(X \mid \mathcal{A})$, we can choose, for any two elements $a, b \in X$, some $\alpha_{a, b}=\alpha_{a, b}(\Pi) \in \mathcal{A}$ so that

$$
2 \alpha_{a, b}=\Pi(a z)+\Pi(b z)-\Pi(a b)
$$

holds and then consider the map

$$
\Pi_{(z)}:\left(\begin{array}{c}
X \\
\leq 2
\end{array}\right) \rightarrow \mathcal{A}
$$

defined by

$$
\Pi_{(z)}(a b):= \begin{cases}0, & \text { if } z \in\{a, b\} \\ \Pi(a z), & \text { if } a=b \neq z, \\ \alpha_{a, b}, & \text { else. }\end{cases}
$$

Clearly, by definition, we have $\Pi_{(z)} \in \mathcal{P}_{\leq 2}(X, z \mid \mathcal{A})$ for every $\Pi \in \mathcal{P}_{(2)}(X \mid \mathcal{A})$. Moreover, we have also

$$
\begin{aligned}
\left(\Pi_{(z)}\right)^{(2)}(a b) & =\Pi_{(z)}(a)+\Pi_{(z)}(b)-2 \Pi_{(z)}(a b) \\
& =\Pi(a z)+\Pi(b z)-2 \alpha_{a, b} \\
& =\Pi(a z)+\Pi(b z)-(\Pi(a z)+\Pi(b z)-\Pi(a b)) \\
& =\Pi(a b)
\end{aligned}
$$

for any two distinct elements $a, b \in X-z$, we have

$$
\left(\Pi_{(z)}\right)^{(2)}(a)=0=\Pi(a)
$$

anyway for all $a \in X$, and we have

$$
\left(\Pi_{(z)}\right)^{(2)}(a z)=\Pi_{(z)}(a)+\Pi_{(z)}(z)-2 \Pi_{(z)}(a z)=\Pi_{(z)}(a)=\Pi(a z)
$$

for all $a \in X$. So, we have found at least one map in $\mathcal{P}_{\leq 2}(X, z \mid \mathcal{A})$, viz., the map $\Pi_{(z)}$, that is mapped by $\zeta_{2}$ onto the element $\Pi \in \mathcal{P}_{(2)}(X \mid \mathcal{A})$, thus implying our claim. 
Fact 3.9. Given any $\Pi \in \mathcal{P}_{\leq 2}(X \mid \mathcal{A})$, one has $\Pi^{(3)} \in \mathcal{P}_{(3)}(X \mid \mathcal{A})$ implying that, in particular, the group homomorphism

$$
\omega_{3}: \mathcal{P}_{\leq 2}(X \mid \mathcal{A}) \rightarrow \mathcal{P}_{(3)}(X \mid \mathcal{A}): \Pi \mapsto \Pi^{(3)}
$$

and its two restrictions

$$
\pi_{3}: \mathcal{P}_{(2)}(X \mid \mathcal{A}) \rightarrow \mathcal{P}_{(3)}(X \mid \mathcal{A}): \Pi \mapsto \Pi^{(3)}
$$

and

$$
\zeta_{3}: \mathcal{P}_{\leq 2}(X, z \mid \mathcal{A}) \rightarrow \mathcal{P}_{(3)}(X \mid \mathcal{A}): \Pi \mapsto \Pi^{(3)}
$$

are well defined.

Indeed, recalling that

$$
\Pi^{(3)}(a b c)=\Pi(a)+\Pi(b)+\Pi(c)-\Pi(a b)-\Pi(b c)-\Pi(c a)
$$

holds for all $a, b, c \in X$ or, equivalently,

$$
\Pi^{(3)}\left(\left\{a_{i}: i \in I\right\}\right)=\sum_{i \in I} \Pi\left(\left\{a_{i}\right\}\right)-\sum_{J \in\left(\begin{array}{l}
I \\
2
\end{array}\right)} \Pi\left(\left\{a_{j}: j \in J\right\}\right)
$$

for any family $\left(a_{i}\right)_{i \in I}$ of elements in $X$ indexed by a 3 -subset $I$, one has

$$
\Pi^{(3)}(a)=\Pi(a)+\Pi(a)+\Pi(a)-\Pi(a)-\Pi(a)-\Pi(a)=0
$$

for every $a \in X$ and, for all $a_{1}, a_{2}, a_{3}, a_{4} \in X$, one has

$$
\begin{aligned}
\sum_{I \in\left(\begin{array}{l}
\{1,2,3,4\} \\
3
\end{array}\right)} \Pi^{(3)}\left(\left\{a_{i}: i \in I\right\}\right) & =\sum_{I \in\left(\begin{array}{c}
\{1,2,3,4\} \\
3
\end{array}\right)}\left(\sum_{i \in I} \Pi\left(\left\{a_{i}\right\}\right)-\sum_{J \in\left(\begin{array}{l}
I \\
2
\end{array}\right)} \Pi\left(\left\{a_{j}: j \in J\right\}\right)\right) \\
& =3 \sum_{i \in\{1,2,3,4\}} \Pi\left(\left\{a_{i}\right\}\right)-2 \sum_{J \in\left(\begin{array}{c}
\{1,2,3,4\} \\
2
\end{array}\right)} \Pi\left(\left\{a_{j}: j \in J\right\}\right)
\end{aligned}
$$

(as there exist exactly three subsets $I \in\left(\begin{array}{c}\{1,2,3,4\} \\ 3\end{array}\right)$ with $i \in I$ for every $i$ in $\{1,2,3,4\}$, and exactly two subsets $I \in\left(\begin{array}{c}\{1,2,3,4\} \\ 3\end{array}\right)$ with $J \in\left(\begin{array}{l}I \\ 2\end{array}\right)$ for every subset $\left.J \in\left(\begin{array}{c}\{1,2,3,4\} \\ 2\end{array}\right)\right)$ as well as

$$
\begin{aligned}
\sum_{J \in\left(\begin{array}{c}
\{1,2,3,4\} \\
2
\end{array}\right)} \Pi^{(3)}\left(\left\{a_{j}: j \in J\right\}\right) & =\sum_{J \in\left(\begin{array}{c}
\{1,2,3,4\} \\
2
\end{array}\right)}\left(\sum_{i \in J} \Pi\left(\left\{a_{i}\right\}\right)-2 \Pi\left(\left\{a_{j}: j \in J\right\}\right)\right) \\
& =3 \sum_{i \in\{1,2,3,4\}} \Pi\left(\left\{a_{i}\right\}\right)-2 \sum_{J \in\left(\begin{array}{c}
\{1,2,3,4\} \\
2
\end{array}\right)} \Pi\left(\left\{a_{j}: j \in J\right\}\right)
\end{aligned}
$$

(as there exist exactly three subsets $J \in\left(\begin{array}{c}\{1,2,3,4\} \\ 2\end{array}\right)$ with $i$ in $J$ for every $\left.i \in\{1,2,3,4\}\right)$ and, therefore, also

$$
\sum_{I \in\left(\begin{array}{c}
\{1,2,3,4\} \\
3
\end{array}\right)} \Pi^{(3)}\left(\left\{a_{i}: i \in I\right\}\right)=\sum_{J \in\left(\begin{array}{c}
\{1,2,3,4\} \\
2
\end{array}\right)} \Pi^{(3)}\left(\left\{a_{j}: j \in J\right\}\right),
$$

as required. 
Fact 3.10. Given any $\Pi \in \mathcal{P}_{\leq 2}(X \mid \mathcal{A})$, one has $\Pi^{(\text {bil })} \in \mathcal{L}_{2}(X \mid \mathcal{A})$ implying that, in particular, the group homomorphism

$$
\omega_{\text {bil }}: \mathcal{P}_{\leq 2}(X \mid \mathcal{A}) \rightarrow \mathcal{L}_{2}(X \mid \mathcal{A}): \Pi \mapsto \Pi^{(\text {bil })}
$$

and its two restrictions

$$
\pi_{\mathrm{bil}}: \mathscr{P}_{(2)}(X \mid \mathcal{A}) \rightarrow \mathcal{L}_{2}(X \mid \mathcal{A}): \Pi \mapsto \Pi^{(\mathrm{bil})}
$$

and

$$
\zeta_{\text {bil }}: \mathscr{P}_{\leq 2}(X, z \mid \mathcal{A}) \rightarrow \mathcal{L}_{2}(X \mid \mathcal{A}): \Pi \mapsto \Pi^{(\text {bil })}
$$

are well defined.

Indeed, given any five elements $x, y, u, v, w \in X$, one has

$$
\Pi^{(\mathrm{bil})}(x y: u v)=\Pi(x v)+\Pi(y u)-\Pi(x u)-\Pi(y v)=\Pi^{(\mathrm{bil})}(u v: x y)
$$

as well as

$$
\begin{aligned}
\Pi^{(\mathrm{bil})}(x y: u v)+\Pi^{(\mathrm{bil})}(x y: v w)= & (\Pi(x v)+\Pi(y u)-\Pi(x u)-\Pi(y v)) \\
& +(\Pi(x w)+\Pi(y v)-\Pi(x v)-\Pi(y w)) \\
= & \Pi(y u)-\Pi(x u)+\Pi(x w)-\Pi(y w) \\
= & \Pi^{(\mathrm{bil})}(x y: u w),
\end{aligned}
$$

for every $\Pi \in \mathcal{P}_{\leq 2}(X \mid \mathcal{A})$, as claimed.

Fact 3.11. Given any $\Sigma \in \mathcal{S}^{*}(X \mid \mathcal{A})$, one has $\Sigma^{(z)} \in \mathcal{P}_{\leq 2}(X, z \mid \mathcal{A})$ implying that the group homomorphism

$$
\sigma_{z}: \mathcal{S}^{*}(X \mid \mathcal{A}) \rightarrow \mathcal{P}_{\leq 2}(X, z \mid \mathcal{A}): \Sigma \mapsto \Sigma^{(z)}
$$

is well defined.

Indeed, given any element $a \in X$, one has

$$
\Sigma^{(z)}(a z)=\Sigma_{+}(a z \mid z)=0
$$

as required.

Fact 3.12. Given any $\Sigma \in \mathcal{S}^{*}(X \mid \mathcal{A})$, one has

$$
\left(\Sigma^{(z)}\right)^{(2)}=\Sigma^{(2)}, \quad\left(\Sigma^{(z)}\right)^{(3)}=\Sigma^{(3)}, \quad \text { and } \quad\left(\Sigma^{(z)}\right)^{(\mathrm{bil})}=\Sigma^{(\mathrm{bil})},
$$

implying (in view of $3.1,3.9$, and 3.10 ) that

$$
\Sigma^{(2)} \in \mathcal{P}_{(2)}(X \mid \mathcal{A}), \quad \Sigma^{(3)} \in \mathcal{P}_{(3)}(X \mid \mathcal{A}), \quad \text { and } \quad \Sigma^{(\mathrm{bil})} \in \mathcal{L}_{2}(X \mid \mathcal{A})
$$


holds for every $\Sigma \in \mathcal{S}^{*}(X \mid \mathcal{A})$, that, in consequence, we have well defined group homomorphisms

$$
\begin{aligned}
& \sigma_{2}: \mathcal{S}^{*}(X \mid \mathcal{A}) \rightarrow \mathcal{P}_{\leq 2}(X, z \mid \mathcal{A}): \Sigma \mapsto \Sigma^{(2)}, \\
& \sigma_{3}: \mathcal{S}^{*}(X \mid \mathcal{A}) \rightarrow \mathcal{P}_{(3)}(X \mid \mathcal{A}): \Sigma \mapsto \Sigma^{(3)},
\end{aligned}
$$

and

$$
\sigma_{\text {bil }}: \mathcal{S}^{*}(X \mid \mathcal{A}) \rightarrow \mathcal{L}_{2}(X \mid \mathcal{A}): \Sigma \mapsto \Sigma^{(\text {bil })}
$$

and that

$$
\sigma_{2}=\zeta_{2} \circ \sigma_{z}, \quad \sigma_{3}=\zeta_{3} \circ \sigma_{z}, \quad \text { and } \quad \sigma_{\text {bil }}=\zeta_{\text {bil }} \circ \sigma_{z}
$$

hold.

Indeed, given any map $\Sigma \in \mathcal{S}^{*}(X \mid \mathcal{A})$ and elements $a, b, c, x, y, u, v \in X$, one has

$$
\begin{aligned}
& \left(\Sigma^{(z)}\right)^{(2)}(a b)=\Sigma^{(z)}(a)+\Sigma^{(z)}(b)-2 \Sigma^{(z)}(a b) \\
& =\Sigma_{+}(a \mid z)+\Sigma_{+}(b \mid z)-2 \Sigma_{+}(a b \mid z) \\
& =\left(\Sigma_{+}(a \mid z)-\Sigma_{+}(a b \mid z)\right)+\left(\Sigma_{+}(b \mid z)-\Sigma_{+}(a b \mid z)\right) \\
& =\Sigma_{+}(a \mid z b)+\Sigma_{+}(b \mid z a) \\
& =\Sigma_{+}(a \mid b) \\
& =\Sigma^{(2)}(a b) \\
& \left(\Sigma^{(z)}\right)^{(3)}(a b c)=\Sigma^{(z)}(a)+\Sigma^{(z)}(b)+\Sigma^{(z)}(c)-\Sigma^{(z)}(a b)-\Sigma^{(z)}(b c)-\Sigma^{(z)}(c a) \\
& =\Sigma_{+}(a \mid z)+\Sigma_{+}(b \mid z)+\Sigma_{+}(c \mid z)-\Sigma_{+}(a b \mid z)-\Sigma_{+}(b c \mid z)-\Sigma_{+}(c a \mid z) \\
& =\left(\Sigma_{+}(a \mid z)-\Sigma_{+}(a b \mid z)\right)+\left(\Sigma_{+}(b \mid z)-\Sigma_{+}(b c \mid z)\right) \\
& +\left(\Sigma_{+}(c \mid z)-\Sigma_{+}(c a \mid z)\right) \\
& =\Sigma_{+}(a \mid b z)+\Sigma_{+}(b \mid c z)+\Sigma_{+}(c \mid a z) \\
& =\left(\Sigma_{+}(a c \mid b z)+\Sigma_{+}(a \mid c b z)\right)+\left(\Sigma_{+}(a b \mid c z)+\Sigma_{+}(b \mid a c z)\right) \\
& +\left(\Sigma_{+}(b c \mid a z)+\Sigma_{+}(c \mid b a z)\right) \\
& =\left(\Sigma_{+}(a z \mid b c)+\Sigma_{+}(a \mid c b z)\right)+\left(\Sigma_{+}(b z \mid c a)+\Sigma_{+}(b \mid c a z)\right) \\
& +\left(\Sigma_{+}(c z \mid a b)+\Sigma_{+}(c \mid a b z)\right) \\
& =\Sigma_{+}(a \mid b c)+\Sigma_{+}(b \mid c a)+\Sigma_{+}(c \mid a b) \\
& =\Sigma^{(3)}(a b c) \text {, }
\end{aligned}
$$


and

$$
\begin{aligned}
& \left(\Sigma^{(z)}\right)^{(\mathrm{bil})}(x y: u v)=\Sigma^{(z)}(x v)+\Sigma^{(z)}(y u)-\Sigma^{(z)}(x u)-\Sigma^{(z)}(y v) \\
& =\Sigma_{+}(x v \mid z)+\Sigma_{+}(y u \mid z)-\Sigma_{+}(x u \mid z)-\Sigma_{+}(y v \mid z) \\
& =\left(\Sigma_{+}(x v \mid z)-\Sigma_{+}(x u \mid z)\right)+\left(\Sigma_{+}(y u \mid z)\right)-\Sigma_{+}(y v \mid z) \\
& =\left(\Sigma_{+}(x v \mid u z)-\Sigma_{+}(x u \mid v z)\right)+\left(\Sigma_{+}(y u \mid v z)-\Sigma_{+}(y v \mid u z)\right) \\
& =\left(\Sigma_{+}(x v \mid u z)-\Sigma_{+}(y v \mid u z)\right)+\left(\Sigma_{+}(y u \mid v z)-\Sigma_{+}(x u \mid v z)\right) \\
& =\left(\Sigma_{+}(x v \mid y u z)-\Sigma_{+}(y v \mid x u z)\right)+\left(\Sigma_{+}(y u \mid x v z)-\Sigma_{+}(x u \mid y v z)\right) \\
& =\left(\Sigma_{+}(x v \mid y u z)+\Sigma_{+}(x v z \mid y u)\right)-\left(\Sigma_{+}(x u z \mid y v)+\Sigma_{+}(x u \mid y v z)\right) \\
& =\Sigma_{+}(x v \mid y u)-\Sigma_{+}(x u \mid y v) \\
& =\Sigma^{(\mathrm{bil})}(x y: u v) \text {, }
\end{aligned}
$$

as claimed.

Fact 3.13. The group homomorphism

$$
\sigma_{z}: S^{*}(X \mid \mathcal{A}) \rightarrow \mathcal{P}_{\leq 2}(X, z \mid \mathcal{A}): \Sigma \mapsto \Sigma^{(z)}
$$

is split surjective provided the set $X$ is finite.

Denoting, for any subset $A$ of $X$, the split $\{A, X-A\}$ by $S_{A}$, we may associate a map $\Sigma_{\Pi} \in \mathcal{S}^{*}(X \mid \mathcal{A})$ to every map $\Pi$ in $\mathcal{P}_{\leq 2}(X, z \mid \mathcal{A})$ by putting

$$
\Sigma_{\Pi}\left(S_{\{a, b\}}\right):=\Pi(a b)
$$

for any two distinct elements $a, b \in X-z$ (if any two such elements exist, i.e., if $\# X \geq 3$ holds),

$$
\Sigma_{\Pi}\left(S_{\{a\}}\right):=\Pi(a)-\sum_{b \in\{X-\{a, z\}\}} \Pi(a b)
$$

for every $a \in X-\{z\}$, and $\Sigma_{\Pi}(S):=0_{\mathcal{A}}$ for every other split $S$ in $S(X)$. As this construction is linear in $\Pi$ (i.e., as $\Sigma_{\Pi+\Pi^{\prime}}=\Sigma_{\Pi}+\Sigma_{\Pi^{\prime}}$ holds for any two maps $\Pi, \Pi^{\prime} \in$ $\left.\mathcal{P}_{(2)}(X \mid \mathcal{A})\right)$, it gives rise to a group homomorphism

$$
\zeta_{s}: \mathcal{P}_{\leq 2}(X, z \mid \mathcal{A}) \rightarrow \mathcal{S}^{*}(X \mid \mathcal{A}): \Pi \mapsto \zeta_{s}(\Pi):=\Sigma_{\Pi},
$$

and it is easy to check that the image

$$
\mathcal{S}_{2}^{*}(X, z \mid \mathcal{A}):=\zeta_{s}\left(\mathscr{P}_{\leq 2}(X, z \mid \mathcal{A})\right)
$$

of $\zeta_{s}$ consists of all maps $\Sigma \in S^{*}(X \mid \mathcal{A})$ whose support is contained in the subset $S_{2}^{*}(X, z)$ of $S(X)$ consisting of all splits $S$ of $X$ for which the subset $S(z)$ in $X$ containing $z$ has co-cardinality 1 or 2 (which happens to be the set $S^{*}(X)$ consisting of all 
proper splits of $X$, i.e., all splits $\{A, B\}$ of $X$ with $A, B \neq \emptyset$, exactly in case $\# X \leq 3$ ) and that

$$
\sigma_{z} \circ \zeta_{s}=\operatorname{Id}_{\mathcal{P}_{\leq 2}(X, z \mid \mathcal{A})}
$$

(as well as $\left.\zeta_{s} \circ \sigma_{z}\right|_{\mathcal{S}_{2}^{*}(X, z \mid \mathcal{A})}=\operatorname{Id}_{\mathcal{S}_{2}^{*}(X, z \mid \mathcal{A})}$ ) holds indeed. In other words, restricting $\sigma_{z}$ to the subgroup $\mathcal{S}_{2}^{*}(X, z \mid \mathcal{A})$ of $\mathcal{S}^{*}(X \mid \mathcal{A})$ yields an isomorphism from $\mathcal{S}_{2}^{*}(X, z \mid \mathcal{A})$ onto $\mathcal{P}_{\leq 2}(X, z \mid \mathcal{A})$ whose inverse is given by the group homomorphism $\zeta_{s}-$ in particular, $\sigma_{z}$ itself is actually a group isomorphism in case $\# X \leq 3$.

Fact 3.14. One has $\Psi^{(2)} \in \mathcal{P}_{(2)}(X \mid \mathcal{A})$ and $\Psi^{(z)} \in \mathcal{P}_{\leq 2}(X, z \mid \mathcal{A})$ for every $\Psi$ in $\mathcal{P}_{(3)}(X \mid$ $\mathcal{A})$, implying that we have well defined group homomorphisms

$$
\psi_{2}: \mathcal{P}_{(3)}(X \mid \mathcal{A}) \rightarrow \mathcal{P}_{(2)}(X \mid \mathcal{A}): \Psi \mapsto \Psi^{(2)}
$$

and

$$
\psi_{z}: \mathcal{P}_{(3)}(X \mid \mathcal{A}) \rightarrow \mathcal{P}_{\leq 2}(X, z \mid \mathcal{A}): \Psi \mapsto \Psi^{(z)} .
$$

Indeed, given any element $a \in X$, one has

$$
\Psi^{(z)}(a z)=\Psi(a z)-\Psi(a z)=0
$$

and

$$
\Psi^{(2)}(a)=\Psi(a)=0
$$

for every $\Psi \in \mathcal{P}_{(3)}(X \mid \mathcal{A})$. And, given any three elements $a, b, c \in X$, one has

$$
\begin{aligned}
\Psi^{(2)}(a b)+\Psi^{(2)}(b c)+\Psi^{(2)}(c a) & =\Psi(a b)+\Psi(b c)+\Psi(c a) \\
& =2 \Psi(a b c) \\
& \in 2 \mathcal{A}
\end{aligned}
$$

for every $\Psi \in \mathcal{P}_{(3)}(X \mid \mathcal{A})$.

Fact 3.15. One has $\Lambda^{(2)} \in \mathcal{P}_{(2)}(X \mid \mathcal{A})$ and $\Lambda^{(z)} \in \mathcal{P}_{\leq 2}(X, z \mid \mathcal{A})$ for every $\Lambda$ in $\mathcal{L}_{2}(X \mid$ $\mathcal{A})$, implying that we have well defined group homomorphisms

$$
\lambda_{2}: \mathcal{L}_{2}(X \mid \mathcal{A}) \rightarrow \mathcal{P}_{(2)}(X \mid \mathcal{A}): \Lambda \mapsto \Lambda^{(2)}
$$

and

$$
\lambda_{z}: \mathcal{L}_{2}(X \mid \mathcal{A}) \rightarrow \mathcal{P}_{\leq 2}(X, z \mid \mathcal{A}): \Lambda \mapsto \Lambda^{(z)}
$$

Indeed, given any element $a \in X$, one has

$$
\Lambda^{(z)}(a z)=\Lambda(a z: z z)=0
$$

and

$$
\Lambda^{(2)}(a)=\Lambda(a a: a a)=0
$$


for every $\Lambda \in \mathcal{L}_{2}(X \mid \mathcal{A})$. And, given any three elements $a, b, c \in X$, one has

$$
\begin{aligned}
\Lambda^{(2)}(a b)+\Lambda^{(2)}(b c)+\Lambda^{(2)}(c a)= & \Lambda(a b: b a)+\Lambda(b c: c b)+\Lambda(c a: a c) \\
= & \Lambda(a b: b c)+\Lambda(a b: c a)+\Lambda(b c: c a) \\
& +\Lambda(b c: a b)+\Lambda(c a: a b)+\Lambda(c a: b c) \\
= & 2 \Lambda(a b: b c)+2 \Lambda(c a: b c)+2 \Lambda(a b: c a) \\
\in & 2 \mathcal{A},
\end{aligned}
$$

for every $\Lambda \in \mathcal{L}_{2}(X \mid \mathcal{A})$.

Fact 3.16. One has

$$
\left(\Pi^{(z)}\right)^{(3)}=\Pi^{(3)} \text { and }\left(\Pi^{(z)}\right)^{(\mathrm{bil})}=\Pi^{(\mathrm{bil})}
$$

for all $\Pi \in \mathcal{P}_{(2)}(X \mid \mathcal{A})$,

$$
\left(\Psi^{(z)}\right)^{(2)}=\Psi^{(2)}
$$

for all $\Psi \in \mathcal{P}_{(3)}(X \mid \mathcal{A})$, and

$$
\left(\Lambda^{(z)}\right)^{(2)}=\Lambda^{(2)}
$$

for all $\Lambda \in \mathcal{L}_{2}(X \mid \mathcal{A})$.

Indeed, given any three elements $a, b, c \in X$, one has

$$
\begin{aligned}
\left(\Pi^{(z)}\right)^{(3)}(a b c)= & \Pi^{(z)}(a)+\Pi^{(z)}(b)+\Pi^{(z)}(c)-\Pi^{(z)}(a b)-\Pi^{(z)}(b c)-\Pi^{(z)}(c a) \\
= & -2 \Pi(a z)-2 \Pi(b z)-2 \Pi(c z)-(\Pi(a b)-\Pi(a z)-\Pi(b z)) \\
& -(\Pi(b c)-\Pi(b z)-\Pi(c z))-(\Pi(c a)-\Pi(c z)-\Pi(a z)) \\
= & -\Pi(a b)-\Pi(b c)-\Pi(c a) \\
= & \Pi^{(3)}(a b c)
\end{aligned}
$$

and, given any four elements $x, y, u, v \in X$, one has

$$
\begin{aligned}
\left(\Pi^{(z)}\right)^{(\mathrm{bil})}(x y: u v)= & \Pi^{(z)}(x v)+\Pi^{(z)}(y u)-\Pi^{(z)}(x u)-\Pi^{(z)}(y v) \\
= & (\Pi(x v)-\Pi(x z)-\Pi(v z))+(\Pi(y u)-\Pi(y z)-\Pi(u z)) \\
& -(\Pi(x u)-\Pi(x z)-\Pi(u z))-(\Pi(y v)-\Pi(y z)-\Pi(v z)) \\
= & \Pi(x v)+\Pi(y u)-\Pi(x u)-\Pi(y v) \\
= & \Pi^{(\mathrm{bil})}(x y: u v)
\end{aligned}
$$


for all $\Pi \in \mathcal{P}_{(2)}(X \mid \mathcal{A})$. And, given any two elements $a, b \in X$, one has

$$
\begin{aligned}
\left(\Psi^{(z)}\right)^{(2)}(a b) & =\Psi^{(z)}(a)+\Psi^{(z)}(b)-2 \Psi^{(z)}(a b) \\
& =\Psi(a z)+\Psi(b z)-2 \Psi(a b z)+2 \Psi(a b) \\
& =\Psi(a z)+\Psi(b z)-(\Psi(a b)+\Psi(a z)+\Psi(b z))+2 \Psi(a b) \\
& =\Psi(a b) \\
& =\Psi^{(2)}(a b)
\end{aligned}
$$

for all $\Psi \in \mathcal{P}_{(3)}(X \mid \mathcal{A})$, and

$$
\begin{aligned}
\left(\Lambda^{(z)}\right)^{(2)}(a b) & =\Lambda^{(z)}(a)+\Lambda^{(z)}(b)-2 \Lambda^{(z)}(a b) \\
& =\Lambda(a z: z a)+\Lambda(b z: z b)-2 \Lambda(a z: z b) \\
& =(\Lambda(a z: z a)-\Lambda(a z: z b))+(\Lambda(b z: z b)-\Lambda(a z: z b)) \\
& =\Lambda(a z: b a)+\Lambda(b a: z b) \\
& =\Lambda(a b: z a)+\Lambda(a b: b z) \\
& =\Lambda(a b: b a) \\
& =\Lambda^{(2)}(a b)
\end{aligned}
$$

for all $\Lambda \in \mathcal{L}_{2}(X \mid \mathcal{A})$, as required.

Fact 3.17. Given any $\Sigma \in \mathcal{S}^{*}(X \mid \mathcal{A})$, one has $\left(\Sigma^{(3)}\right)^{(z)}=\left(\Sigma^{(\mathrm{bil})}\right)^{(z)}=\Sigma^{(z)}$, i.e., one has

$$
\psi_{z} \circ \sigma_{3}=\lambda_{z} \circ \sigma_{\text {bil }}=\sigma_{z}
$$

In particular, one has

$$
\psi_{z} \circ \zeta_{3}=\lambda_{z} \circ \zeta_{\text {bil }}=\operatorname{Id}_{\mathcal{P}_{\leq 2}(X, z \mid \mathcal{A})} .
$$

Indeed, given any two elements $a, b \in X$, one has

$$
\begin{aligned}
\left(\Sigma^{(3)}\right)^{(z)}(a b) & =\Sigma^{(3)}(a b z)-\Sigma^{(3)}(a b) \\
& =\Sigma_{+}(a b \mid z)+\Sigma_{+}(a \mid b z)+\Sigma_{+}(b \mid a z)-\Sigma_{+}(a \mid b) \\
& =\Sigma_{+}(a b \mid z) \\
& =\Sigma^{(z)}(a b)
\end{aligned}
$$


as well as

$$
\begin{aligned}
\left(\Sigma^{(\mathrm{bil})}\right)^{(z)}(a b) & =\Sigma^{(\mathrm{bil})}(a z: z b) \\
& =\Sigma_{+}(a b \mid z) \\
& =\Sigma^{(z)}(a b)
\end{aligned}
$$

while the last assertion follows from the fact that the above identities imply that

$$
\left(\psi_{z} \circ \zeta_{3}\right) \circ \sigma_{z}=\psi_{z} \circ\left(\zeta_{3} \circ \sigma_{z}\right)=\psi_{z} \circ \sigma_{3}=\sigma_{z}
$$

as well as

$$
\left(\lambda_{z} \circ \zeta_{\mathrm{bil}}\right) \circ \sigma_{z}=\lambda_{z} \circ\left(\zeta_{\mathrm{bil}} \circ \sigma_{z}\right)=\lambda_{z} \circ \sigma_{\mathrm{bil}}=\sigma_{z}
$$

holds. So, if $X$ is finite, the surjectivity of $\sigma_{z}$ implies that also

$$
\psi_{z} \circ \zeta_{3}=\lambda_{z} \circ \zeta_{\text {bil }}=\operatorname{Id}_{\mathcal{P}_{\leq 2}(X, z \mid \mathcal{A})}
$$

must hold, as claimed. However, even if $X$ is infinite, the same must hold in view of the fact that, to establish this identity, one only needs to establish that $\left(\Pi^{(3)}\right)^{(z)}(a b)=$ $\left(\Pi^{(\mathrm{bil})}\right)^{(z)}(a b)=\Pi(a b)$ holds for all $\Pi \in \mathcal{P}_{\leq 2}(X, z \mid \mathcal{A})$ and any two elements $a, b \in$ $X$, and this involves only finitely many elements from $X$. So, the claim must hold for any set if it holds for every finite set.

Fact 3.18. Given any $\Psi \in \mathcal{P}_{(3)}(X \mid \mathcal{A})$, one has $\left(\Psi^{(z)}\right)^{(3)}=\Psi$, i.e., one has also $\zeta_{3} \circ \psi_{z}=\operatorname{Id}_{\mathcal{P}_{(3)}(X \mid \mathcal{A})}$, that is, the two group homomorphisms $\zeta_{3}$ and $\psi_{z}$ are mutually inverse group isomorphisms, implying that $\sigma_{3}: \mathcal{S}^{*}(X \mid \mathcal{A}) \rightarrow \mathcal{P}_{(3)}(X \mid \mathcal{A})$ is also a split-surjective group homomorphisms.

Indeed, given three elements $a, b, c \in X$, one has

$$
\begin{aligned}
\left(\Psi^{(z)}\right)^{(3)}(a b c)= & \Psi^{(z)}(a)+\Psi^{(z)}(b)+\Psi^{(z)}(c)-\Psi^{(z)}(a b)-\Psi^{(z)}(b c)-\Psi^{(z)}(c a) \\
= & \Psi(a z)+\Psi(b z)+\Psi(c z)-(\Psi(a b z)-\Psi(a b)) \\
& -(\Psi(b c z)-\Psi(b c))-(\Psi(c a z)-\Psi(c a)) \\
= & (\Psi(a z)+\Psi(b z)+\Psi(c z)+\Psi(a b)+\Psi(b c)+\Psi(c a)) \\
& -\Psi(a b z)-\Psi(b c z)-\Psi(c a z) \\
= & (\Psi(a b z)+\Psi(b c z)+\Psi(c a z)+\Psi(a b c)) \\
& -\Psi(a b z)-\Psi(b c z)-\Psi(c a z) \\
= & \Psi(a b c),
\end{aligned}
$$


for every $\Psi \in \mathcal{P}_{(3)}(X \mid \mathcal{A})$. In consequence, one has also

$$
\begin{aligned}
\sigma_{3} \circ\left(\zeta_{s} \circ \psi_{z}\right) & =\left(\zeta_{3} \circ \sigma_{z}\right) \circ\left(\zeta_{s} \circ \psi_{z}\right) \\
& =\zeta_{3} \circ\left(\sigma_{z} \circ \zeta_{s}\right) \circ \psi_{z} \\
& =\zeta_{3} \circ \psi_{z} \\
& =\operatorname{Id}_{\mathcal{P}_{(3)}(X \mid \mathcal{A})},
\end{aligned}
$$

implying that $\sigma_{3}$ is also split-surjective, as claimed.

Fact 3.19. Given any $\Lambda \in \mathcal{L}_{2}(X \mid \mathcal{A})$, one has $\left(\Lambda^{(z)}\right)^{(\text {bil })}=\Lambda$, i.e., one has also $\zeta_{\text {bil }} \circ$ $\lambda_{z}=\operatorname{Id}_{\mathcal{L}_{2}(X \mid \mathcal{A})}$, that is, also the two group homomorphisms $\zeta_{\text {bil }}$ and $\lambda_{z}$ are mutually inverse group isomorphisms and $\sigma_{\text {bil }}: \mathcal{S}^{*}(X \mid \mathcal{A}) \rightarrow \mathcal{L}_{2}(X \mid \mathcal{A})$ is also split-surjective.

Indeed, given any four elements $x, y, u, v \in X$, one has

$$
\begin{aligned}
\left(\Lambda^{(z)}\right)^{(\text {bil })}(x y: u v) & =\Lambda^{(z)}(x v)+\Lambda^{(z)}(y u)-\Lambda^{(z)}(x u)-\Lambda^{(z)}(y v) \\
& =\Lambda(x z: z v)+\Lambda(y z: z u)-\Lambda(x z: z u)-\Lambda(y z: z v) \\
& =(\Lambda(x z: u z)+\Lambda(x z: z v))+(\Lambda(z y: u z)+\Lambda(z y: z v)) \\
& =\Lambda(x z: u v)+\Lambda(z y: u v) \\
& =\Lambda(x y: u v)
\end{aligned}
$$

for every $\Lambda \in \mathcal{L}_{2}(X \mid \mathcal{A})$ and, therefore, also

$$
\begin{aligned}
\sigma_{\text {bil }} \circ\left(\zeta_{s} \circ \lambda_{z}\right) & =\left(\zeta_{\text {bil }} \circ \sigma_{z}\right) \circ\left(\zeta_{s} \circ \lambda_{z}\right) \\
& =\zeta_{\text {bil }} \circ\left(\sigma_{z} \circ \zeta_{s}\right) \circ \lambda_{z} \\
& =\zeta_{\text {bil }} \circ \lambda_{z} \\
& =\operatorname{Id}_{\mathcal{P}_{(3)}(X \mid \mathcal{A})},
\end{aligned}
$$

implying that also $\sigma_{\text {bil }}$ is split-surjective, as claimed.

Fact 3.20. Given any $\Sigma \in \mathcal{S}^{*}(X \mid \mathcal{A})$, one has

$$
\Sigma^{(3)}=\left(\Sigma^{(\mathrm{bil})}\right)^{(3)} \text { and } \quad \Sigma^{(\mathrm{bil})}=\left(\Sigma^{(3)}\right)^{(\mathrm{bil})} .
$$

In consequence, writing any $\Psi \in \mathcal{P}_{(3)}(X \mid \mathcal{A})$ in the form

$$
\Psi=\left(\psi_{z}(\Psi)\right)^{(3)}=\left(\sigma_{z}\left(\zeta_{s}\left(\psi_{z}(\Psi)\right)\right)\right)^{(3)}=\left(\zeta_{s}\left(\psi_{z}(\Psi)\right)\right)^{(3)}
$$

(cf. 3.18, 3.13, and 3.12), and any $\Lambda \in \mathcal{L}_{2}(X \mid \mathcal{A})$ in the form

$$
\Lambda=\left(\lambda_{z}(\Lambda)\right)^{(\mathrm{bil})}=\left(\sigma_{\mathrm{z}}\left(\zeta_{s}\left(\lambda_{z}(\Lambda)\right)\right)\right)^{(\mathrm{bil})}=\left(\zeta_{s}\left(\lambda_{z}(\Lambda)\right)\right)^{(\mathrm{bil})}
$$


(cf. 3.19, 3.13, and 3.12), one can see that

$$
\Psi^{(\mathrm{bil})}=\left(\left(\zeta_{s}\left(\Psi_{z}(\Psi)\right)\right)^{(3)}\right)^{(\mathrm{bil})}=\left(\zeta_{s}\left(\Psi_{z}(\Psi)\right)\right)^{(\mathrm{bil})} \in \mathcal{L}_{2}(X \mid \mathcal{A})
$$

and

$$
\Lambda^{(3)}=\left(\left(\zeta_{s}\left(\lambda_{z}(\Lambda)\right)\right)^{(\mathrm{bil})}\right)^{(3)}=\left(\zeta_{s}\left(\lambda_{z}(\Lambda)\right)\right)^{(3)} \in \mathcal{P}_{(3)}(X \mid \mathcal{A})
$$

holds for all $\Psi \in \mathcal{P}_{(3)}(X \mid \mathcal{A})$ and $\Lambda \in \mathcal{L}_{2}(X \mid \mathcal{A})$. In particular, also the two group homomorphisms

$$
\psi_{\text {bil }}: \mathcal{P}_{(3)}(X \mid \mathcal{A}) \rightarrow \mathcal{L}_{2}(X \mid \mathcal{A}): \Psi \rightarrow \Psi^{(\mathrm{bil})}
$$

and

$$
\lambda_{3}: \mathcal{L}_{2}(X \mid \mathcal{A}) \rightarrow \mathcal{P}_{(3)}(X \mid \mathcal{A}): \Lambda \rightarrow \Lambda^{(3)}
$$

are well-defined, and we have

$$
\lambda_{3} \circ \sigma_{\text {bil }}=\sigma_{3}=\zeta_{3} \circ \sigma_{z}=\zeta_{3} \circ \lambda_{z} \circ \sigma_{\text {bil }}
$$

as well as

$$
\psi_{\text {bil }} \circ \sigma_{3}=\sigma_{\text {bil }}=\zeta_{\text {bil }} \circ \sigma_{z}=\zeta_{\text {bil }} \circ \psi_{z} \circ \sigma_{3}
$$

and, therefore, in view of the surjectivity of $\sigma_{\text {bil }}$ and $\sigma_{3}$ in case $X$ is finite, also

$$
\lambda_{3}=\zeta_{3} \circ \lambda_{z} \text { and } \psi_{\text {bil }}=\zeta_{\text {bil }} \circ \psi_{z}
$$

Indeed, given three elements $a, b, c \in X$, one has

$$
\begin{aligned}
\left(\Sigma^{(\mathrm{bil})}\right)^{(3)}(a b c) & =\Sigma^{(\mathrm{bil})}(a b: b c)+\Sigma^{(\mathrm{bil})}(b c: c a)+\Sigma^{(\mathrm{bil})}(c a: a b) \\
& =\Sigma_{+}(c a \mid b)+\Sigma_{+}(a b \mid c)+\Sigma_{+}(b c \mid a) \\
& =\Sigma^{(3)}(a b c)
\end{aligned}
$$

and, given any four elements $x, y, u, v \in X$, one has

$$
\begin{aligned}
\left(\Sigma^{(3)}\right)^{(\text {bil })}(x y: u v) & =\Sigma^{(3)}(x y v)-\Sigma^{(3)}(x v)-\left(\Sigma^{(3)}(x y u)-\Sigma^{(3)}(x u)\right) \\
& =\left(\Sigma^{(3)}\right)^{(y)}(x v)-\left(\Sigma^{(3)}\right)^{(y)}(x u) \\
& =\Sigma_{+}(x v \mid y)-\Sigma_{+}(x u \mid y) \\
& =\Sigma_{+}(x v \mid y u)-\Sigma_{+}(x u \mid y v) \\
& =\Sigma^{(\text {bil })}(x y: u v),
\end{aligned}
$$

as claimed.

So, all the group homomorphisms we have been referring to in the diagram in Theorem 1.5 have now been shown to be well-defined (that is, they have been shown to associate, to any map in their domain, a map in the (sub)group that was specified 
as their range). We have seen also that the two pairs of anti-parallel solid arrows in the much sparser diagram

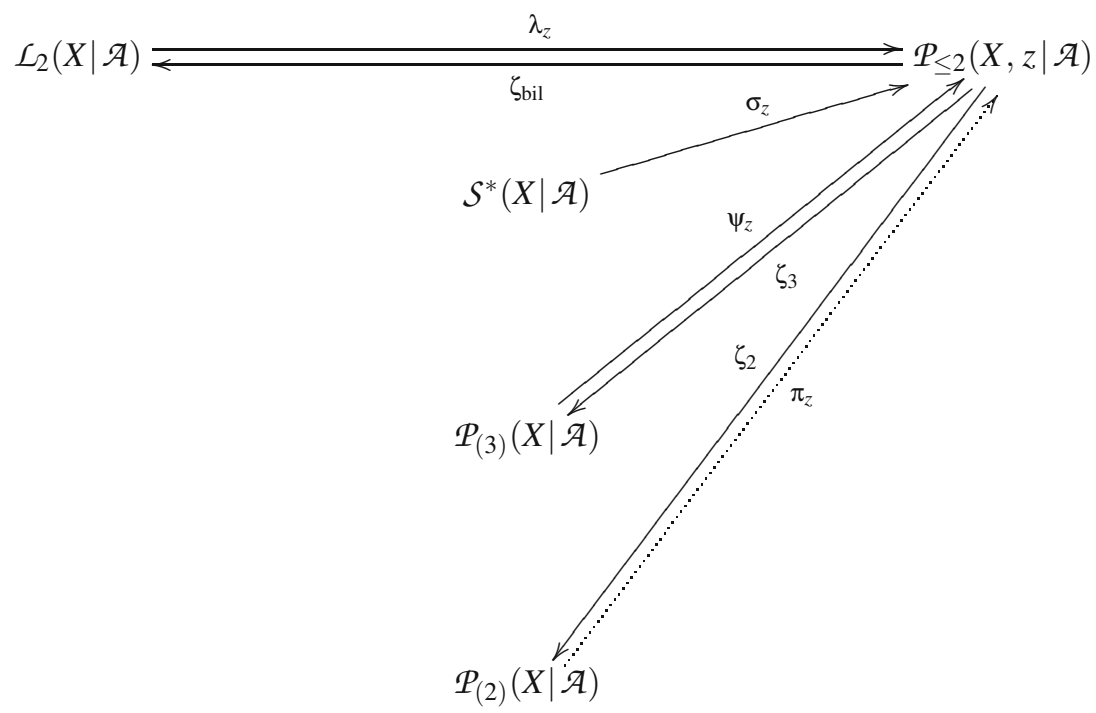

represent mutually inverse group homomorphisms while, composing the homomorphisms $\pi_{z}$ represented by the dotted arrow with its anti-parallel counterpart $\zeta_{2}$ in either one of the two possible orders, one obtains -2 times the identity homomorphisms of either one of the two groups $\mathcal{P}_{(2)}(X \mid \mathcal{A})$ or $\mathcal{P}_{\leq 2}(X, z \mid \mathcal{A})$, respectively. Thus, it remains to observe that, for any one of the remaining group homomorphisms

$$
\gamma=\gamma_{\mathcal{G}_{1} \rightarrow \mathcal{G}_{2}}: \mathcal{G}_{1} \rightarrow \mathcal{G}_{2}
$$

from either one of the groups $\mathcal{G}_{1}=\mathcal{S}^{*}(X \mid \mathcal{A}), \mathcal{P}_{(2)}(X \mid \mathcal{A}), \mathcal{P}_{(3)}(X \mid \mathcal{A})$, or $\mathcal{L}_{2}(X \mid \mathcal{A})$ into either one of the groups $\mathcal{G}_{2}=\mathcal{P}_{(2)}(X \mid \mathcal{A}), \mathcal{P}_{(3)}(X \mid \mathcal{A})$, or $\mathcal{L}_{2}(X \mid \mathcal{A})$ represented by an arrow in the diagram in Theorem 1.5 , not yet represented by an arrow in the sparse diagram above, one has

$$
\gamma=\gamma_{\mathcal{P}_{\leq 2}(X, z \mid \mathcal{A}) \rightarrow \mathcal{G}_{2}} \circ \gamma_{\mathcal{G}_{1} \rightarrow \mathcal{P}_{\leq 2}(X, z \mid \mathcal{A})}
$$

for the unique group homomorphisms represented by an arrow in that sparse diagram from $\mathcal{G}_{1}$ into $\mathcal{P}_{\leq 2}(X, z \mid \mathcal{A})$ and from $\mathcal{P}_{\leq 2}(X, z \mid \mathcal{A})$ into $\mathcal{G}_{2}$. However, we have done this already above in 3.12 in case $\mathcal{G}_{1}:=\mathcal{S}^{*}(X \mid \mathcal{A})$, in 3.16 in case $\mathcal{G}_{1}:=\mathcal{P}_{(2)}(X \mid \mathcal{A})$ and $\mathcal{G}_{2}:=\mathcal{P}_{(3)}(X \mid \mathcal{A})$ or $\mathcal{G}_{2}:=\mathcal{L}_{2}(X \mid \mathcal{A})$, and in case $\mathcal{G}_{1}:=\mathcal{P}_{(3)}(X \mid \mathcal{A})$ or $\mathcal{G}_{1}:=$ $\mathcal{L}_{2}(X \mid \mathcal{A})$ and $\mathcal{G}_{2}:=\mathcal{P}_{(2)}(X \mid \mathcal{A})$, and in the two remaining cases $\mathcal{G}_{1}:=\mathcal{P}_{(3)}(X \mid \mathcal{A})$ and $\mathcal{G}_{2}:=\mathcal{L}_{2}(X \mid \mathcal{A})$, and $\mathcal{G}_{1}:=\mathcal{L}_{2}(X \mid \mathcal{A})$ and $\mathcal{G}_{2}:=\mathcal{P}_{(3)}(X \mid \mathcal{A})$ in 3.20. So, taking all things together, we have indeed established Theorem 1.5.

\section{Abelian Groups Without 2-Torsion}

Now, assume that $\mathcal{A}$ does not contain any element of order 2 and note that this implies that 
- the group homomorphisms $\pi_{z}, \pi_{3}, \pi_{\mathrm{bil}}, \zeta_{2}, \psi_{2}$, and $\lambda_{2}$ are injective group homomorphisms,

- $\zeta_{2}$ induces an isomorphism from $\mathcal{P}_{\leq 2}(X, z \mid \mathcal{A})$ onto $\mathcal{P}_{(2)}(X \mid \mathcal{A})$ whose inverse is given by the (then well-defined!) map

$$
\pi_{z}^{*}:=-\frac{1}{2} \pi_{z}: \mathcal{P}_{(2)}(X \mid \mathcal{A}) \rightarrow \mathcal{P}_{\leq 2}(X, z \mid \mathcal{A}): \Pi \mapsto-\frac{1}{2} \Pi^{(z)}
$$

where, for every map $\Pi \in \mathcal{P}_{(2)}(X \mid \mathcal{A})$ and all $x, y \in X$, the image $-\frac{1}{2} \Pi^{(z)}(x y)$ of the subset $\{x, y\} \in\left(\begin{array}{c}X \\ \leq 2\end{array}\right)$ with respect to the map $\pi_{z}^{*}(\Pi)=-\frac{1}{2} \Pi^{(z)}$ in $\mathcal{P}_{\leq 2}(X, z \mid \mathcal{A})$ is given by

$$
-\frac{1}{2} \Pi^{(z)}(x y)=\frac{1}{2}(\Pi(x z)+\Pi(y z)-\Pi(x y)),
$$

a group element that coincides with

$$
\begin{aligned}
\frac{1}{2}\left(\Sigma_{+}(x \mid z)+\Sigma_{+}(y \mid z)-\Sigma_{+}(x \mid y)\right)= & \frac{1}{2}\left(\Sigma_{+}(x y \mid z)+\Sigma_{+}(x \mid y z)+\Sigma_{+}(x y \mid z)\right. \\
& \left.+\Sigma_{+}(y \mid x z)-\Sigma_{+}(z x \mid y)-\Sigma_{+}(x \mid y z)\right) \\
= & \Sigma_{+}(x y \mid z) \\
= & \Sigma^{(z)}(x y)
\end{aligned}
$$

in case $\Pi=\Sigma^{(2)}$ holds for some map $\Sigma \in \mathcal{S}^{*}(X \mid \mathcal{A})$,

- $\psi_{2}$ induces an isomorphism from $\mathcal{P}_{(3)}(X \mid \mathcal{A})$ onto $\mathcal{P}_{(2)}(X \mid \mathcal{A})$ whose inverse is given by the (then also well-defined!) map

$$
\pi_{3}^{*}:=-\frac{1}{2} \pi_{3}: \mathcal{P}_{(2)}(X \mid \mathcal{A}) \rightarrow \mathcal{P}_{(3)}(X \mid \mathcal{A}): \Pi \mapsto-\frac{1}{2} \Pi^{(3)}
$$

where, for every map $\Pi \in \mathcal{P}_{(2)}(X \mid \mathcal{A})$ and all $a, b, c \in X$, the image $-\frac{1}{2} \Pi^{(3)}(a b c)$ of the subset $\{a, b, c\} \in\left(\begin{array}{c}X \\ \leq 3\end{array}\right)$ with respect to the map $\pi_{3}^{*}(\Pi)=-\frac{1}{2} \Pi^{(3)}$ in $\mathcal{P}_{(3)}(X \mid$ $\mathcal{A})$ is given by

$$
-\frac{1}{2} \Pi^{(3)}(a b c)=\frac{1}{2}(\Pi(a b)+\Pi(b c)+\Pi(c a))
$$

a group element that coincides with

$$
\begin{aligned}
\frac{1}{2}(\Pi(a b)+\Pi(b c)+\Pi(c a)) & =\frac{1}{2}\left(\Sigma_{+}(a \mid b)+\Sigma_{+}(b \mid c)+\Sigma_{+}(c \mid a)\right) \\
& =\Sigma_{+}(a \mid b c)+\Sigma_{+}(b \mid c a)+\Sigma_{+}(c \mid a b) \\
& =\Sigma^{(3)}(a b c)
\end{aligned}
$$

in case $\Pi=\Sigma^{(2)}$ holds for some map $\Sigma \in \mathcal{S}^{*}(X \mid \mathcal{A})$, 
- and $\lambda_{2}$ induces an isomorphism from $\mathcal{L}_{2}(X \mid \mathcal{A})$ onto $\mathcal{P}_{(2)}(X \mid \mathcal{A})$ whose inverse is given by the (then also well-defined!) group homomorphism

$$
\pi_{\mathrm{bil}}^{*}:=-\frac{1}{2} \pi_{\mathrm{bil}}: \mathcal{P}_{(2)}(X \mid \mathcal{A}) \rightarrow \mathcal{L}_{2}(X \mid \mathcal{A}): \Pi \mapsto-\frac{1}{2} \Pi^{((\mathrm{bil})}
$$

where, for every map $\Pi \in \mathcal{P}_{(2)}(X \mid \mathcal{A})$ and all $x, y, u, v \in X$, the image $-\frac{1}{2} \Pi^{(\text {bil })}(x y$ : $u v)$ of the pair $((x, y),(u, v))$ in $\mathcal{G}(X)^{2}$ with respect to the map $\pi_{\text {bil }}^{*}(\Pi)=-\frac{1}{2} \Pi^{(\text {bil })}$ is given by

$$
-\frac{1}{2} \pi_{\mathrm{bil}}(x y: u v)=\frac{1}{2}(\Pi(x u)+\Pi(y v)-\Pi(x v)-\Pi(y u)),
$$

a group element that coincides with

$$
\frac{1}{2}\left(\Sigma_{+}(x \mid u)+\Sigma_{+}(y \mid v)-\Sigma_{+}(x \mid v)-\Sigma_{+}(y \mid u)\right)
$$

in case $\Pi=\Sigma^{(2)}$ holds for some map $\Sigma \in \mathcal{S}^{*}(X \mid \mathcal{A})$ and hence, in view of

$$
\begin{aligned}
& \Sigma_{+}(x \mid v)=\Sigma_{+}(x y u \mid v)+\Sigma_{+}(x y \mid u v)+\Sigma_{+}(x u \mid y v)+\Sigma_{+}(x \mid y u v), \\
& \Sigma_{+}(y \mid u)=\Sigma_{+}(x y v \mid u)+\Sigma_{+}(x y \mid u v)+\Sigma_{+}(y v \mid x u)+\Sigma_{+}(y \mid x u v), \\
& \Sigma_{+}(x \mid u)=\Sigma_{+}(x y v \mid u)+\Sigma_{+}(x y \mid u v)+\Sigma_{+}(x v \mid y u)+\Sigma_{+}(x \mid y u v), \\
& \Sigma_{+}(y \mid v)=\Sigma_{+}(x y u \mid v)+\Sigma_{+}(x y \mid u v)+\Sigma_{+}(y u \mid x v)+\Sigma_{+}(y \mid x u v),
\end{aligned}
$$

with

$$
\Sigma_{+}(x v \mid y u)-\Sigma_{+}(x u \mid y v)=(x y: u v)_{\Sigma}=\Lambda_{\Sigma}(x y: u v) .
$$

So, we have

$$
\pi_{z}^{*} \circ \zeta_{2}=\operatorname{Id}_{\mathcal{P}_{\leq 2}(X, z \mid \mathcal{A})}, \quad \pi_{3}^{*} \circ \psi_{2}=\operatorname{Id}_{\mathcal{P}_{(3)}(X \mid \mathcal{A})}, \quad \pi_{\mathrm{bil}}^{*} \circ \lambda_{2}=\operatorname{Id}_{\mathcal{L}_{2}(X \mid \mathcal{A})},
$$

as well as

$$
\zeta_{2} \circ \pi_{z}^{*}=\psi_{2} \circ \pi_{3}^{*}=\lambda_{2} \circ \pi_{\mathrm{bil}}^{*}=\operatorname{Id}_{\mathcal{P}_{(2)}(X \mid \mathcal{A})},
$$

and

$$
\sigma_{z}=\pi_{z}^{*} \circ \sigma_{2}, \quad \sigma_{3}=\pi_{3}^{*} \circ \sigma_{2}, \quad \sigma_{\text {bil }}=\pi_{\text {bil }}^{*} \circ \sigma_{2}
$$

in this case, implying in particular that also $\mathcal{P}_{(2)}(X \mid \mathcal{A})$ is canonically isomorphic to a direct sum of $\left(\begin{array}{c}\# X \\ 2\end{array}\right)$ copies of $\mathcal{A}$ in case this group does not contain any element of order 2, and that also the group homomorphism $\sigma_{2}$ must be split-surjective in this case.

It follows also that the group homomorphism $\pi_{z}^{*}$ coincides with the celebrated Farris transform (cf. [24, p. 181], [22, pp. 835 ff], and [23, p. 491], see also [17] for further references and a recent survey of uses of this transformation and [16] for further recent applications). The results put together in this note can therefore be viewed as pointing towards yet another way of studying the Farris transform and its variants in a more general context.

The following commutative diagram summarizes our discussion: 
Theorem 4.1. Assume that $\mathcal{A}$ is an abelian group that does not contain any element of order 2. Then, given any fixed element $z \in X$, the group homomorphisms defined above together form the commutative diagram:

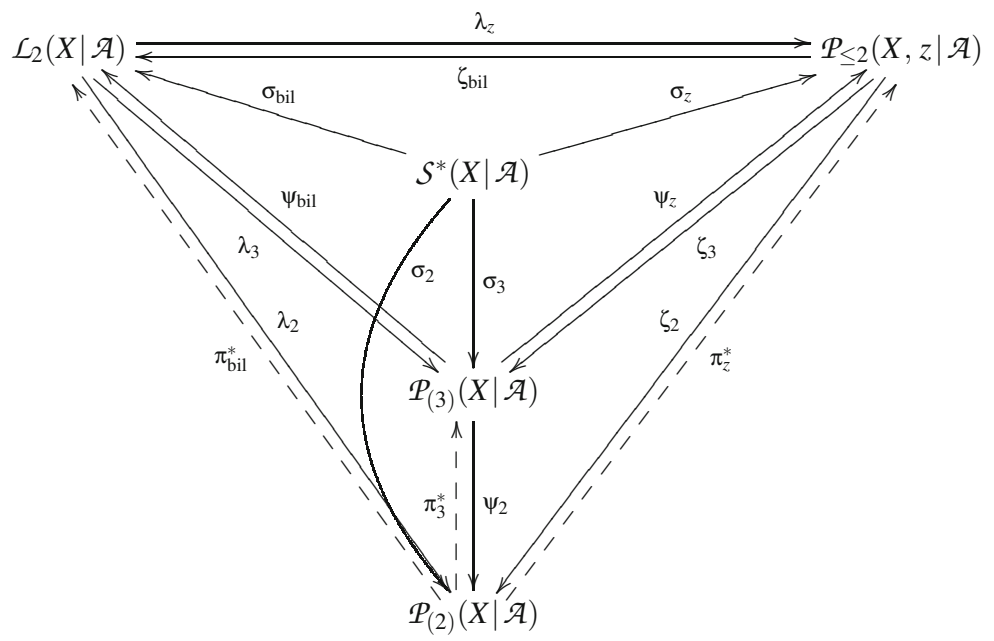

All arrows emanating from the group $\mathcal{S}^{*}(X \mid \mathcal{A})$ in the center of the diagram are splitsurjective provided $X$ is a finite set, all pairs of anti-parallel arrows represent mutually inverse group isomorphisms - in particular, all group homomorphisms inbetween the four "trace groups" $\mathcal{L}_{2}(X \mid \mathcal{A}), \mathcal{P}_{\leq 2}(X, z \mid \mathcal{A}), \mathcal{P}_{(3)}(X \mid \mathcal{A})$, and $\mathcal{P}_{(2)}(X \mid \mathcal{A})$ are group isomorphisms - and the arrows drawn with broken lines represent maps that cannot be defined unless $\mathcal{A}$ satisfies the assumption that it does not contain any elements of order 2.

\section{References}

1. J. Backelin and A.W.M. Dress, The kernel of the split map, in preparation.

2. J. Backelin and S. Linusson, Parity splits by triple point distances in $X$-trees, Ann. Combin. 10 (2006) 1-18.

3. H.-J. Bandelt, Recognition of tree metrics, SIAM J. Discrete Math. 3 (1) (1990) 1-6.

4. H.-J. Bandelt and A.W.M. Dress, A canonical split decomposition theory for metrics on a finite set, Adv. Math. 92 (1) (1992) 47-105.

5. H.-J. Bandelt and A.W.M. Dress, Reconstructing the shape of a tree from observed dissimilarity data, Adv. Appl. Math. 7 (3) (1986) 309-343.

6. H.-J. Bandelt and A.W.M. Dress, Split decomposition: a new and useful approach to phylogenetic analysis of distance data, Mol. Phylogenet Evol. 1 (3) (1992) 242-252.

7. H.-J. Bandelt and A.W.M. Dress, Weak hierarchies associated with similarity measures — an additive clustering technique, Bull. Math. Biol. 51 (1) (1989) 133-166.

8. H.-J. Bandelt and M.A. Steel, Symmetric matrices representable by weighted trees over a cancellative abelian monoid, SIAM J. Discrete Math. 8 (4) (1995) 517-525. 
9. G.M. Barker, Phylogenetic diversity: a quantitative framework for measurement of priority and achievement in biodiversity conservation, Biol. J. Linnean Soc. 76 (2) (2002) 165-194.

10. S. Böcker and A.W.M. Dress, Recovering symbolically dated, rooted trees from symbolic ultrametrics, Adv. Math. 138 (1) (1998) 105-125.

11. H. Colonius and H.H. Schultze, Trees constructed from empirical relations, Braunschweiger Berichte aus dem Institut fuer Psychologie 1, Braunschweig, 1977.

12. C. Devauchelle, A. Dress, A. Grossmann, S. Grünewald, and A. Henaut, Constructing Hierarchical set systems, Ann. Combin. 8 (4) (2004) 441-456.

13. A.W.M. Dress, Split decomposition over an abelian group, part 2: group-valued split systems with weakly compatible support, Discrete Appl. Math. 157 (10) 2349-2360.

14. A.W.M. Dress, Split decomposition over an abelian group, part 3: group-valued split systems with compatible support, Manuscript.

15. A.W.M. Dress and P. Erdös, $X$-trees and weighted quartet systems, Ann. Combin. 7 (2) (2003) 155-169.

16. A.W.M. Dress et al., $\Delta$ additive and $\Delta$ ultra-additive maps, Gromov's trees, and the Farris transform, Discrete Appl. Math. 146 (1) (2005) 51-73.

17. A.W.M. Dress, K. Huber, and V. Moulton, Some uses of the Farris transform in mathematics and phylogenetics — a review, Ann. Combin. 11 (1) (2007) 1-37.

18. A.W.M. Dress and M.A. Steel, Mapping edge sets to splits in trees: the path index and parsimony, Ann. Combin. 10 (1) (2006) 77-96.

19. A.W.M. Dress and M.A. Steel, Phylogenetic diversity over an abelian group, 11 (2) (2007) 143-160.

20. S.N. Evans and T.P. Speed, Invariants of some probability models used in phylogenetic inference, Ann. Statist. 21 (1) (1993) 355-377.

21. D.P. Faith, Conservation evaluation and phylogenetic diversity, Biol. Conserv. 61 (1) (1992) $1-10$.

22. J.S. Farris, On the phenetic approach to vertebrate classification, In: Major Patterns in Vertebrate Evolution, M.K. Hecht, P.C. Goody, and B.M. Hecht, Eds., Plenum Press, New York, (1977) pp. 823-850.

23. J.S. Farris, The information content of the phylogenetic system, Sys. Zool. 28 (4) (1979) 483-519.

24. J.S. Farris, A.G. Kluge, and M.J. Eckardt, A numerical approach to phylogenetic systematics, Sys. Zool. 19 (2) (1970) 172-189.

25. J. Felsenstein, Inferring Phylogenies, Sinauer Press, Sunderland, 2004.

26. W.M. Fitch and E. Margoliash, Construction of phylogenetic trees, Science 155 (1967) 279-284.

27. W.J. Heiser and M. Bennani, Triadic distance models: aximomatization and least squares representation, J. Math. Psych. 41 (2) (1997) 189-206.

28. S. Joly and Le Calvé, Three-way distances, J. Classification 12 (2) (1995) 191-205.

29. B. Korte, L. Lovász, and R. Schrader, Greedoids, Algorithms and Combinatorics, Springer-Verlag, Berlin, 1991.

30. L. Pachter and D. Speyer, Reconstructing trees from subtree weights, Appl. Math. Lett. 17 (6) (2004) 615-621.

31. C. Semple and M.A. Steel, Cyclic permutations and evolutionary trees, Adv. Appl. Math. 32 (4) (2004) 669-680.

32. C. Semple and M.A. Steel, Phylogenetics, Oxford University Press, Oxford, 2003. 
33. M.A. Steel, Phylogenetic diversity and the greedy algorithm, Syst. Biol. 54 (4) (2005) 527-529.

Open Access This article is distributed under the terms of the Creative Commons Attribution Noncommercial License which permits any noncommercial use, distribution, and reproduction in any medium, provided the original author(s) and source are credited. 